Supporting Information for:

\title{
Mechanism and Mitigation of Irreversible Material Loss within Gel-based Single Walled Carbon Nanotube Purification Schemes
}

Watts, Brennan P.t; Rolsma, Calebt; Dolan, Marshalt; and Tvrdy, Kevin†*

tDepartment of Chemistry \& Biochemistry; University of Colorado, Colorado Springs; Colorado Springs, Colorado

*ktvrdy@uccs.edu

\section{Table of contents}

$\underline{\text { Section }} \underline{\text { Title }}$

$\underline{\text { Page }}$

S1. Detailed Materials and Methods .................................................S2

S2. Quantitative results from absorbance spectra deconvolutions generated by and used in this work

S3. Comparative spectral fitting and deconvolution analysis S18

S4. Intermediate data pertaining to the adsorption of SWCNT to Sephacryl at various SDS concentration and elution thereof

S5. Intermediate data pertaining to the adsorption of SWCNT to Sephacryl at various SWCNT concentration and elution thereof

S6. References S25 


\section{S1. Detailed Materials and Methods}

\section{S1.1 Materials and Equipment}

\section{S1.1.1 Hydrogel}

- Sephacryl S-200 High Resolution (Sephacryl S200) - GE Healthcare Bio-Sciences AB; Lot\# 10240334

\section{S1.1.2 Other Chemicals}

- Carbon Nanotubes - Sigma Aldrich; mfr. no. Signis ${ }^{\circledR}$ SG65i; single walled enriched in $(6,5)$ chirality ( $\geq 95 \%$ carbon basis [ $\geq 95 \%$ as carbon nanotubes]); Lot \# MKBZ1159V

- Sodium Dodecyl Sulfate (SDS) - Sigma Life Science; Lot\# SLCH6627; $\geq 98.5 \%$

- Sodium Fluoride (NaF) - JT Baker; Lot \# 951104, 100.2\% (Supplier specified greater than unity purity)

- Sodium Chloride (NaCl) - Fischer; Lot\# 163661, 99.7\%.

- Sodium Bromide ( $\mathrm{NaBr}$ ) - Mallinckrodt; Lot\# 0535 kcdx

- Sodium lodide (Nal) - Fischer; Lot\# 136809A.

- Urea - Fischer; Lot\# 148623, 99\%

- Deionized water - resistivity of $15 \mathrm{M} \Omega$

\section{S1.1.3 Equipment}

- Thermo Scientific Disposable Plastic Columns (Number 29920); $7 \mathrm{~mm}$ inner diameter, $60 \mathrm{~mm}$ height; porous polyethylene disk ( $30 \mu \mathrm{m}$ pore size) used below the packed gel bed

- Tip horn Sonicator - Branson Digital Sonifier, Model 250, 1/2" Tip, coupled with $50 \mathrm{~mL}$ jacketed beaker for temperature stabilization during prolonged sonication

- Ultracentrifuge - Sorvall MX 120 Plus Micro-Ultracentrifuge with S50-ST Rotor

- 120v Digital Tube Revolver; Thermo Scientific

- HPAgilent 8453 G1103A Spectrometer; Agilent Technologies

- VWR 96-Well disposable thermocycling plate for ABI cyclers (200 $\mu \mathrm{L})$ employed as sample holder during Raman analysis of SWCNT fractions

- Raman spectra collected using Nearfield Scanning Optical Microscope (NSOM, Alpha300, Witec) system in Raman mode with Nd-YAG laser $(532 \mathrm{~nm}$ ) excitation, 20x objective lens (NA =0.4), and acquisition times ranging from 1-10 seconds and averaging 10 scans for each spectrum.

\section{S1.2 Methods}

\section{S1.2.1 Generation of SWCNT suspension in aqueous SDS solution}

Aliquots of individualized SWCNT were prepared by tip-horn sonication (20W, 1/2" tip) of suspensions with varied volume but approximate concentration of $1 \mathrm{mg}$ SWCNT powder per $\mathrm{mL}$ of $70 \mathrm{mM}$ SDS solution. The length of sonication was normalized to afford a sonication state (in units of Watt*hours $/ \mathrm{mL}$ ) of 2.8, which is consistent with materials employed in our past works. ${ }^{1-3}$

For this work, Signis SG65i SWCNT powder was employed for its enriched purity of the $(6,5)$ chirality, a feature which affords accurate deconvolution and quantitation of the chiral species present in a sample, while also demonstrating the ability of Sephacryl gel to afford chiral enrichment. To sediment out metal nano-catalysts employed during SWCNT synthesis and isolate individualized nanotubes, sonicated 
aliquots were subjected to ultracentrifugation with RCF between approximately 180,000 and 200,000 for a period of 2 hours. Upon collection of the centrifuged material, the upper $\sim 80-90 \%$ of solution was removed and the lower $10 \%$ delegated to waste.

With the exception of investigations of the behavior of reclaimed SWCNT and reclaimed gel, and kinetic investigations presented in Figure 6 in the main body, all SWCNT suspensions were applied following ultracentrifugation and without additional treatment or purification. However, for the listed experiments suspensions which had previously been enriched by interaction with Sephacryl gel were used. Further details on this methodology are provided in previous works. ${ }^{2}$

\section{S1.2.2 General Procedural Information}

It is important to note that for all experiments performed here multiple passes of a SWCNT suspension through a gel bed were employed. This is because spectroscopy has revealed there exists a significant difference in the SWCNT adsorbed by a gel bed following one pass and two passes; however, the difference between two and more passes is by comparison negligible, and so for the experiments reported here a number of between 2 and 4 passes was applied. For some experiments, greater numbers of passes were used to ensure a SWCNT-saturated state for the gel, and in some cases to offset differences in the amount of time it takes a SWCNT suspension to pass through a gel bed once. 


\section{S2. Quantitative results from absorbance spectra deconvolutions generated by and used in this work}

This work applies methods previously described elsewhere ${ }^{1,2}$ for the deconvolution of SWCNT absorbance spectra and differential absorbance spectroscopy in order to quantify the number and type of SWCNT which are adsorbed to a hydrogel. Briefly, an in-house written Mathematica software was used to identify least squares best-fit traces in energy-space between a given experimental absorbance spectrum and the sum of calculated absorbance profiles (each belonging to a specific chirality with associated peak center), along with an amorphous carbon background. To afford differential absorbance traces (which are descriptive of material adsorbed to or retained by the gel), the difference was taken between two SWCNT absorbance traces, for example that before and after interaction with a gel (to afford a description of adsorbed materials).

The total number of each SWCNT chirality and per-chirality fractional abundances for each SWCNT suspension are reported below and are organized according to the data presented in each figure within the main text. The abbreviations "Ads" and "FT" refer to calculated adsorbed spectra and measured flowthrough spectra, respectively. Grey-highlighted cells represent values pertaining to control suspensions, meaning suspensions of SWCNT which did not interact with hydrogel. Persons interested in using the Mathematica script described here to fit absorbance spectra of SWCNT suspensions are encouraged to contact the corresponding author (Kevin Tvrdy at ktvrdy@uccs.edu), who will freely provide the most updated version of this software.

\begin{tabular}{|c|c|c|c|c|c|c|}
\hline \begin{tabular}{|l|} 
Figure 1 \\
\end{tabular} & \multicolumn{2}{|c|}{ Post-Cent Panel A } & \multicolumn{2}{|c|}{ Control Panel D } & \multicolumn{2}{|c|}{ FT Panel E } \\
\hline Chirality $(n, m)$ & $\# S W N T / m L$ & fractional purity $\#$ & $\# S W N T / m L$ & fractional purity & \#SWNT/mL & fractional purity \\
\hline$(10,2)$ & $8.08 \mathrm{E}+12$ & $5.77 \mathrm{E}-02$ & $2.03 E+12$ & $8.32 \mathrm{E}-02$ & $1.61 \mathrm{E}+12$ & $8.81 \mathrm{E}-02$ \\
\hline$(7,5)$ & $2.79 E+13$ & $1.99 \mathrm{E}-01$ & $6.63 E+12$ & $2.71 \mathrm{E}-01$ & $5.66 \mathrm{E}+12$ & $3.10 \mathrm{E}-01$ \\
\hline$(6,5)$ & $3.37 E+13$ & $2.40 \mathrm{E}-01$ & $6.50 \mathrm{E}+12$ & $2.66 \mathrm{E}-01$ & $4.20 \mathrm{E}+12$ & $2.30 \mathrm{E}-01$ \\
\hline$(7,3)$ & $2.63 \mathrm{E}+13$ & $1.88 \mathrm{E}-01$ & $6.11 \mathrm{E}+12$ & $2.50 \mathrm{E}-01$ & $5.01 \mathrm{E}+12$ & $2.74 \mathrm{E}-01$ \\
\hline$(8,3)$ & $1.47 \mathrm{E}+13$ & $1.05 \mathrm{E}-01$ & $1.21 \mathrm{E}+12$ & $4.98 \mathrm{E}-02$ & $7.34 \mathrm{E}+11$ & $4.02 \mathrm{E}-02$ \\
\hline$(9,1)$ & $9.10 \mathrm{E}+12$ & $6.49 \mathrm{E}-02$ & $6.84 \mathrm{E}+11$ & $2.80 \mathrm{E}-02$ & $6.35 \mathrm{E}+11$ & $3.48 \mathrm{E}-02$ \\
\hline \multirow[t]{2}{*}{$(6,4)$} & $2.03 E+13$ & $1.45 \mathrm{E}-01$ & $1.24 \mathrm{E}+12$ & 5.09E-02 & $4.16 \mathrm{E}+11$ & $2.28 \mathrm{E}-02$ \\
\hline & \multicolumn{2}{|c|}{ Adsorbed Panel E } & \multicolumn{2}{|c|}{ Eluted Panel F } & \multicolumn{2}{|c|}{ Retained Panel G } \\
\hline Chirality $(n, m)$ & $\# S W N T / m L$ & fractional purity & $\# S W N T / m L$ & fractional purity & \begin{tabular}{l|l|l}
$\# S W N T / m L$ & $f$ \\
\end{tabular} & fractional purity \\
\hline$(10,2)$ & $7.65 \mathrm{E}+10$ & $1.28 \mathrm{E}-02$ & $7.65 \mathrm{E}+10$ & $1.28 \mathrm{E}-02$ & $1.26 \mathrm{E}+11$ & 4.49E-02 \\
\hline$(7,5)$ & $8.21 \mathrm{E}+11$ & 1.37E-01 & $8.21 \mathrm{E}+11$ & 1.37E-01 & $2.95 \mathrm{E}+11$ & $1.05 \mathrm{E}-01$ \\
\hline$(6,5)$ & $2.06 \mathrm{E}+12$ & $3.44 \mathrm{E}-01$ & $2.06 \mathrm{E}+12$ & $3.44 \mathrm{E}-01$ & $3.51 \mathrm{E}+11$ & $1.25 \mathrm{E}-01$ \\
\hline$(7,3)$ & $1.23 E+12$ & $2.05 \mathrm{E}-01$ & $1.23 \mathrm{E}+12$ & $2.05 E-01$ & $1.10 \mathrm{E}+12$ & $3.91 \mathrm{E}-01$ \\
\hline$(8,3)$ & $3.72 \mathrm{E}+11$ & $6.20 \mathrm{E}-02$ & $3.72 \mathrm{E}+11$ & $6.20 \mathrm{E}-02$ & $2.80 E+11$ & $9.99 \mathrm{E}-02$ \\
\hline$(9,1)$ & $1.88 \mathrm{E}+11$ & $3.13 \mathrm{E}-02$ & $1.88 \mathrm{E}+11$ & $3.13 E-02$ & $1.48 \mathrm{E}+11$ & $5.26 \mathrm{E}-02$ \\
\hline$(6,4)$ & $1.25 \mathrm{E}+12$ & $2.08 \mathrm{E}-01$ & $1.25 \mathrm{E}+12$ & $2.08 \mathrm{E}-01$ & $5.08 \mathrm{E}+11$ & $1.81 \mathrm{E}-01$ \\
\hline
\end{tabular}

Table S1: SWCNT concentration and relative abundance of each chiral species for data presented in Figure 1 in the main text. 


\begin{tabular}{|c|c|c|c|c|c|c|}
\hline \multicolumn{7}{|l|}{ Figure 2} \\
\hline & \multicolumn{2}{|c|}{26 mM SDS- Control } & \multicolumn{2}{|c|}{$26 \mathrm{mM}$ SDS- FT } & \multicolumn{2}{|c|}{26 mM SDS - Ads } \\
\hline Chirality $(n, m)$ & \#SWNT/mL & fractional purity & \#SWNT/mL & fractional purity & \#SWNT/mL & fractional purity \\
\hline$(10,2)$ & $5.98 \mathrm{E}+11$ & $8.75 \mathrm{E}-02$ & $5.17 \mathrm{E}+11$ & $9.73 \mathrm{E}-02$ & $2.52 \mathrm{E}+10$ & $1.60 \mathrm{E}-02$ \\
\hline$(7,5)$ & $1.83 \mathrm{E}+12$ & $2.68 \mathrm{E}-01$ & $1.56 \mathrm{E}+12$ & $2.94 \mathrm{E}-01$ & $2.48 \mathrm{E}+11$ & $1.57 \mathrm{E}-01$ \\
\hline$(6,5)$ & $1.80 \mathrm{E}+12$ & $2.64 \mathrm{E}-01$ & $1.25 \mathrm{E}+12$ & $2.35 \mathrm{E}-01$ & $5.00 \mathrm{E}+11$ & $3.16 \mathrm{E}-01$ \\
\hline$(7,3)$ & $1.68 \mathrm{E}+12$ & $2.45 \mathrm{E}-01$ & $1.36 \mathrm{E}+12$ & $2.56 \mathrm{E}-01$ & $3.48 \mathrm{E}+11$ & $2.20 \mathrm{E}-01$ \\
\hline$(8,3)$ & $3.91 \mathrm{E}+11$ & $5.73 \mathrm{E}-02$ & $2.77 \mathrm{E}+11$ & $5.21 \mathrm{E}-02$ & $1.10 \mathrm{E}+11$ & 6.96E-02 \\
\hline$(9,1)$ & $1.94 \mathrm{E}+11$ & $2.84 \mathrm{E}-02$ & $1.78 \mathrm{E}+11$ & 3.36E-02 & $5.84 \mathrm{E}+10$ & 3.69E-02 \\
\hline \multirow[t]{2}{*}{$(6,4)$} & $3.41 \mathrm{E}+11$ & $4.98 \mathrm{E}-02$ & $1.68 \mathrm{E}+11$ & $3.15 \mathrm{E}-02$ & $2.91 \mathrm{E}+11$ & 1.84E-01 \\
\hline & \multicolumn{2}{|c|}{26 mM SDS- Elute } & \multicolumn{2}{|c|}{35 mM SDS - Control } & \multicolumn{2}{|c|}{35 mM SDS - FT } \\
\hline Chirality $(n, m)$ & $\# S W N T / m L$ & fractional purity & \#SWNT/mL & fractional purity & \#SWNT/mL & fractional purity \\
\hline$(10,2)$ & $4.99 \mathrm{E}+10$ & 1.40E-02 & $5.98 \mathrm{E}+11$ & 8.85E-02 & $5.30 \mathrm{E}+11$ & 1.02E-01 \\
\hline$(7,5)$ & $5.66 \mathrm{E}+11$ & $1.59 \mathrm{E}-01$ & $1.83 \mathrm{E}+12$ & 2.70E-01 & $1.55 \mathrm{E}+12$ & $2.98 \mathrm{E}-01$ \\
\hline$(6,5)$ & $1.48 \mathrm{E}+12$ & 4.16E-01 & $1.78 \mathrm{E}+12$ & $2.64 \mathrm{E}-01$ & $1.20 \mathrm{E}+12$ & 2.31E-01 \\
\hline$(7,3)$ & $4.53 \mathrm{E}+11$ & $1.27 \mathrm{E}-01$ & $1.64 \mathrm{E}+12$ & $2.42 \mathrm{E}-01$ & $1.33 \mathrm{E}+12$ & $2.56 \mathrm{E}-01$ \\
\hline$(8,3)$ & $2.18 \mathrm{E}+11$ & $6.12 \mathrm{E}-02$ & $3.93 \mathrm{E}+11$ & $5.81 \mathrm{E}-02$ & $2.65 E+11$ & $5.09 \mathrm{E}-02$ \\
\hline$(9,1)$ & $1.14 \mathrm{E}+11$ & $3.20 \mathrm{E}-02$ & $1.90 \mathrm{E}+11$ & $2.82 \mathrm{E}-02$ & $1.79 \mathrm{E}+11$ & 3.43E-02 \\
\hline \multirow[t]{2}{*}{$(6,4)$} & $6.83 \mathrm{E}+11$ & $1.91 \mathrm{E}-01$ & $3.34 \mathrm{E}+11$ & $4.94 \mathrm{E}-02$ & $1.43 \mathrm{E}+11$ & $2.75 \mathrm{E}-02$ \\
\hline & \multicolumn{2}{|c|}{$35 \mathrm{mM}$ SDS- Ads } & \multicolumn{2}{|c|}{35 mM SDS- Elute } & \multicolumn{2}{|c|}{44 mM SDS- Control } \\
\hline Chirality $(n, m)$ & \#SWNT/mL & fractional purity & \#SWNT/mL & fractional purity & \#SWNT/mL & fractional purity \\
\hline$(10,2)$ & $7.91 \mathrm{E}+08$ & 4.91E-04 & $2.49 \mathrm{E}+10$ & 7.57E-03 & $5.89 \mathrm{E}+11$ & $9.05 \mathrm{E}-02$ \\
\hline$(7,5)$ & $2.47 \mathrm{E}+11$ & $1.53 \mathrm{E}-01$ & $4.92 \mathrm{E}+11$ & 1.49E-01 & $1.76 \mathrm{E}+12$ & $2.71 \mathrm{E}-01$ \\
\hline$(6,5)$ & $5.07 \mathrm{E}+11$ & $3.15 \mathrm{E}-01$ & $1.45 \mathrm{E}+12$ & $4.40 \mathrm{E}-01$ & $1.73 E+12$ & 2.67E-01 \\
\hline$(7,3)$ & $3.70 \mathrm{E}+11$ & $2.29 \mathrm{E}-01$ & $3.78 \mathrm{E}+11$ & 1.15E-01 & $1.54 \mathrm{E}+12$ & $2.37 \mathrm{E}-01$ \\
\hline$(8,3)$ & $1.13 \mathrm{E}+11$ & 7.04E-02 & $1.76 \mathrm{E}+11$ & $5.35 \mathrm{E}-02$ & $3.69 E+11$ & 5.67E-02 \\
\hline$(9,1)$ & $5.72 \mathrm{E}+10$ & $3.55 \mathrm{E}-02$ & $9.11 \mathrm{E}+10$ & $2.76 \mathrm{E}-02$ & $1.83 \mathrm{E}+11$ & $2.82 \mathrm{E}-02$ \\
\hline \multirow[t]{2}{*}{$(6,4)$} & $3.16 \mathrm{E}+11$ & 1.96E-01 & $6.81 \mathrm{E}+11$ & 2.07E-01 & $3.22 \mathrm{E}+11$ & $4.96 \mathrm{E}-02$ \\
\hline & \multicolumn{2}{|c|}{44 mM SDS - FT } & \multicolumn{2}{|c|}{$44 \mathrm{mM}$ SDS - Ads } & \multicolumn{2}{|c|}{$44 \mathrm{mM}$ SDS - Elute } \\
\hline Chirality $(n, m)$ & \#SWNT/mL & fractional purity & $\# S W N T / m L$ & fractional purity & \#SWNT/mL & fractional purity \\
\hline$(10,2)$ & $4.89 \mathrm{E}+11$ & 9.96E-02 & $1.94 \mathrm{E}+10$ & $1.21 \mathrm{E}-02$ & $2.84 \mathrm{E}+10$ & 7.97E-03 \\
\hline$(7,5)$ & $1.50 \mathrm{E}+12$ & 3.06E-01 & $2.25 \mathrm{E}+11$ & $1.40 \mathrm{E}-01$ & $5.28 \mathrm{E}+11$ & $1.48 \mathrm{E}-01$ \\
\hline$(6,5)$ & $1.13 \mathrm{E}+12$ & $2.29 \mathrm{E}-01$ & $5.40 \mathrm{E}+11$ & 3.37E-01 & $1.58 \mathrm{E}+12$ & 4.43E-01 \\
\hline$(7,3)$ & $1.27 \mathrm{E}+12$ & $2.58 \mathrm{E}-01$ & $3.21 \mathrm{E}+11$ & $2.00 \mathrm{E}-01$ & $3.98 \mathrm{E}+11$ & $1.12 \mathrm{E}-01$ \\
\hline$(8,3)$ & $2.41 \mathrm{E}+11$ & 4.91E-02 & $1.12 \mathrm{E}+11$ & 6.98E-02 & $1.79 \mathrm{E}+11$ & 5.02E-02 \\
\hline$(9,1)$ & $1.71 \mathrm{E}+11$ & 3.47E-02 & $5.34 \mathrm{E}+10$ & 3.33E-02 & $8.86 \mathrm{E}+10$ & 2.48E-02 \\
\hline \multirow[t]{2}{*}{$(6,4)$} & $1.11 \mathrm{E}+11$ & $2.26 \mathrm{E}-02$ & $3.31 \mathrm{E}+11$ & 2.07E-01 & $7.62 \mathrm{E}+11$ & $2.14 \mathrm{E}-01$ \\
\hline & \multicolumn{2}{|c|}{$53 \mathrm{mM}$ SDS - Control } & \multicolumn{2}{|c|}{$53 \mathrm{mM}$ SDS - FT } & \multicolumn{2}{|c|}{$53 \mathrm{mM}$ SDS- Ads } \\
\hline Chirality $(n, m)$ & $\# S W N T / m L$ & fractional purity & \#SWNT/mL & fractional purity & \#SWNT/mL & fractional purity \\
\hline$(10,2)$ & $4.88 \mathrm{E}+11$ & 7.57E-02 & $4.41 \mathrm{E}+11$ & $9.41 \mathrm{E}-02$ & $4.34 \mathrm{E}+07$ & 2.32E-05 \\
\hline$(7,5)$ & $1.77 \mathrm{E}+12$ & $2.75 \mathrm{E}-01$ & $1.49 \mathrm{E}+12$ & 3.17E-01 & $2.50 \mathrm{E}+11$ & 1.34E-01 \\
\hline$(6,5)$ & $1.78 \mathrm{E}+12$ & $2.77 \mathrm{E}-01$ & $9.75 \mathrm{E}+11$ & $2.08 \mathrm{E}-01$ & $7.44 \mathrm{E}+11$ & $3.98 \mathrm{E}-01$ \\
\hline$(7,3)$ & $1.50 \mathrm{E}+12$ & $2.33 \mathrm{E}-01$ & $1.28 \mathrm{E}+12$ & $2.73 \mathrm{E}-01$ & $2.53 \mathrm{E}+11$ & 1.36E-01 \\
\hline$(8,3)$ & $3.78 \mathrm{E}+11$ & $5.87 \mathrm{E}-02$ & $2.65 \mathrm{E}+11$ & 5.66E-02 & $1.36 \mathrm{E}+11$ & 7.30E-02 \\
\hline$(9,1)$ & $1.85 \mathrm{E}+11$ & $2.87 \mathrm{E}-02$ & $1.86 \mathrm{E}+11$ & 3.97E-02 & $6.02 \mathrm{E}+10$ & 3.22E-02 \\
\hline$(6,4)$ & $3.40 \mathrm{E}+11$ & $5.27 \mathrm{E}-02$ & $5.32 \mathrm{E}+10$ & 1.14E-02 & $4.26 \mathrm{E}+11$ & $2.28 \mathrm{E}-01$ \\
\hline
\end{tabular}




\begin{tabular}{|c|c|c|c|c|c|c|}
\hline \multirow[b]{2}{*}{ Chirality $(n, m)$} & \multicolumn{2}{|c|}{$53 \mathrm{mM}$ SDS - Elute } & \multicolumn{2}{|c|}{$61 \mathrm{mM}$ SDS- Control } & \multicolumn{2}{|c|}{$61 \mathrm{mM}$ SDS - FT } \\
\hline & \#SWNT/mL & fractional purity & \#SWNT/mL & fractional purity & $\# S W N T / m L$ & fractional purity \\
\hline$(10,2)$ & $9.51 \mathrm{E}+09$ & $2.42 \mathrm{E}-03$ & $5.21 \mathrm{E}+11$ & $7.98 \mathrm{E}-02$ & $4.38 \mathrm{E}+11$ & $9.09 \mathrm{E}-02$ \\
\hline$(7,5)$ & $4.81 \mathrm{E}+11$ & $1.22 \mathrm{E}-01$ & $1.79 \mathrm{E}+12$ & $2.74 \mathrm{E}-01$ & $1.53 \mathrm{E}+12$ & $3.18 \mathrm{E}-01$ \\
\hline$(6,5)$ & $1.92 \mathrm{E}+12$ & 4.89E-01 & $1.82 \mathrm{E}+12$ & $2.78 \mathrm{E}-01$ & $1.07 \mathrm{E}+12$ & 2.22E-01 \\
\hline$(7,3)$ & $3.77 \mathrm{E}+11$ & $9.58 \mathrm{E}-02$ & $1.49 \mathrm{E}+12$ & $2.28 \mathrm{E}-01$ & $1.26 \mathrm{E}+12$ & $2.61 \mathrm{E}-01$ \\
\hline$(8,3)$ & $1.32 \mathrm{E}+11$ & 3.35E-02 & $3.83 E+11$ & 5.87E-02 & $2.88 \mathrm{E}+11$ & 5.96E-02 \\
\hline$(9,1)$ & $6.92 \mathrm{E}+10$ & $1.76 \mathrm{E}-02$ & $1.87 \mathrm{E}+11$ & $2.86 \mathrm{E}-02$ & $1.95 \mathrm{E}+11$ & 4.05E-02 \\
\hline \multirow[t]{2}{*}{$(6,4)$} & $9.40 \mathrm{E}+11$ & 2.39E-01 & $3.43 E+11$ & $5.25 \mathrm{E}-02$ & $4.24 \mathrm{E}+10$ & 8.78E-03 \\
\hline & \multicolumn{2}{|c|}{$61 \mathrm{mM}$ SDS - Ads } & \multicolumn{2}{|c|}{$61 \mathrm{mM}$ SDS - Elute } & \multicolumn{2}{|c|}{70 mM SDS - Control } \\
\hline Chirality $(n, m)$ & \#SWNT/mL & fractional purity & \#SWNT/mL & fractional purity & \#SWNT/mL & fractional purity \\
\hline$(10,2)$ & $5.29 \mathrm{E}+07$ & $3.03 \mathrm{E}-05$ & $3.54 \mathrm{E}+09$ & 9.49E-04 & $5.09 \mathrm{E}+11$ & $7.83 \mathrm{E}-02$ \\
\hline$(7,5)$ & $2.19 \mathrm{E}+11$ & $1.25 \mathrm{E}-01$ & $4.35 \mathrm{E}+11$ & 1.17E-01 & $1.80 \mathrm{E}+12$ & $2.77 \mathrm{E}-01$ \\
\hline$(6,5)$ & $6.74 \mathrm{E}+11$ & $3.86 \mathrm{E}-01$ & $1.86 \mathrm{E}+12$ & $4.98 \mathrm{E}-01$ & $1.84 \mathrm{E}+12$ & $2.83 \mathrm{E}-01$ \\
\hline$(7,3)$ & $2.60 \mathrm{E}+11$ & $1.49 \mathrm{E}-01$ & $3.04 \mathrm{E}+11$ & 8.17E-02 & $1.47 \mathrm{E}+12$ & $2.26 \mathrm{E}-01$ \\
\hline$(8,3)$ & $1.18 \mathrm{E}+11$ & 6.77E-02 & $1.11 \mathrm{E}+11$ & $2.98 \mathrm{E}-02$ & $3.62 E+11$ & 5.57E-02 \\
\hline$(9,1)$ & $5.28 \mathrm{E}+10$ & $3.03 \mathrm{E}-02$ & $5.26 \mathrm{E}+10$ & $1.41 \mathrm{E}-02$ & $1.80 \mathrm{E}+11$ & $2.77 \mathrm{E}-02$ \\
\hline \multirow[t]{2}{*}{$(6,4)$} & $4.23 \mathrm{E}+11$ & $2.42 \mathrm{E}-01$ & $9.64 \mathrm{E}+11$ & $2.58 \mathrm{E}-01$ & $3.39 \mathrm{E}+11$ & $5.21 \mathrm{E}-02$ \\
\hline & \multicolumn{2}{|c|}{70 mM SDS - FT } & \multicolumn{2}{|c|}{70 mM SDS - Ads } & \multicolumn{2}{|c|}{$70 \mathrm{mM}$ SDS - Elute } \\
\hline Chirality $(n, m)$ & \#SWNT/mL & fractional purity & \#SWNT/mL & fractional purity & $\# S W N T / m L$ & fractional purity \\
\hline$(10,2)$ & $3.88 \mathrm{E}+11$ & 8.04E-02 & $2.02 \mathrm{E}+08$ & $1.25 \mathrm{E}-04$ & $2.05 E+09$ & 6.47E-04 \\
\hline$(7,5)$ & $1.55 \mathrm{E}+12$ & $3.21 \mathrm{E}-01$ & $2.09 \mathrm{E}+11$ & $1.29 \mathrm{E}-01$ & $3.23 \mathrm{E}+11$ & 1.02E-01 \\
\hline$(6,5)$ & $1.15 \mathrm{E}+12$ & 2.39E-01 & $6.37 \mathrm{E}+11$ & 3.93E-01 & $1.57 \mathrm{E}+12$ & 4.93E-01 \\
\hline$(7,3)$ & $1.20 \mathrm{E}+12$ & $2.49 \mathrm{E}-01$ & $2.30 \mathrm{E}+11$ & $1.42 \mathrm{E}-01$ & $2.49 \mathrm{E}+11$ & $7.85 \mathrm{E}-02$ \\
\hline$(8,3)$ & $3.09 \mathrm{E}+11$ & 6.39E-02 & $7.48 \mathrm{E}+10$ & $4.61 \mathrm{E}-02$ & $7.07 \mathrm{E}+10$ & $2.23 \mathrm{E}-02$ \\
\hline$(9,1)$ & $2.05 \mathrm{E}+11$ & $4.25 \mathrm{E}-02$ & $3.76 \mathrm{E}+10$ & $2.32 \mathrm{E}-02$ & $3.05 E+10$ & 9.61E-03 \\
\hline \multirow[t]{2}{*}{$(6,4)$} & $2.11 \mathrm{E}+10$ & 4.37E-03 & $4.31 \mathrm{E}+11$ & 2.66E-01 & $9.33 \mathrm{E}+11$ & $2.94 \mathrm{E}-01$ \\
\hline & \multicolumn{2}{|c|}{79 mM SDS - Control } & \multicolumn{2}{|c|}{$79 \mathrm{mM}$ SDS - FT } & \multicolumn{2}{|c|}{79 mM SDS - Ads } \\
\hline Chirality $(n, m)$ & \#SWNT/mL & fractional purity & \#SWNT/mL & fractional purity & $\# S W N T / m L$ & fractional purity \\
\hline$(10,2)$ & $5.25 \mathrm{E}+11$ & 7.96E-02 & $3.69 \mathrm{E}+11$ & 7.67E-02 & $5.56 \mathrm{E}+09$ & 3.30E-03 \\
\hline$(7,5)$ & $1.83 \mathrm{E}+12$ & $2.78 \mathrm{E}-01$ & $1.56 \mathrm{E}+12$ & $3.23 E-01$ & $2.27 \mathrm{E}+11$ & $1.35 \mathrm{E}-01$ \\
\hline$(6,5)$ & $1.88 \mathrm{E}+12$ & $2.85 \mathrm{E}-01$ & $1.18 \mathrm{E}+12$ & $2.46 \mathrm{E}-01$ & $6.35 \mathrm{E}+11$ & 3.77E-01 \\
\hline$(7,3)$ & $1.46 \mathrm{E}+12$ & $2.21 \mathrm{E}-01$ & $1.18 \mathrm{E}+12$ & $2.45 \mathrm{E}-01$ & $2.43 \mathrm{E}+11$ & $1.44 \mathrm{E}-01$ \\
\hline$(8,3)$ & $3.68 \mathrm{E}+11$ & $5.59 \mathrm{E}-02$ & $3.07 \mathrm{E}+11$ & $6.38 \mathrm{E}-02$ & $8.57 \mathrm{E}+10$ & $5.08 \mathrm{E}-02$ \\
\hline$(9,1)$ & $1.83 \mathrm{E}+11$ & $2.78 \mathrm{E}-02$ & $2.02 \mathrm{E}+11$ & $4.20 \mathrm{E}-02$ & $4.50 \mathrm{E}+10$ & 2.67E-02 \\
\hline \multirow[t]{2}{*}{$(6,4)$} & $3.46 \mathrm{E}+11$ & $5.25 \mathrm{E}-02$ & $1.25 \mathrm{E}+10$ & $2.59 \mathrm{E}-03$ & $4.44 \mathrm{E}+11$ & $2.63 \mathrm{E}-01$ \\
\hline & \multicolumn{2}{|c|}{$79 \mathrm{mM}$ SDS - Elute } & \multicolumn{2}{|c|}{$88 \mathrm{mM}$ SDS - Control } & \multicolumn{2}{|c|}{$88 \mathrm{mM}$ SDS - FT } \\
\hline Chirality $(n, m)$ & \#SWNT/mL & fractional purity & \#SWNT/mL & fractional purity & $\# S W N T / m L$ & fractional purity \\
\hline$(10,2)$ & $2.90 \mathrm{E}+10$ & $9.90 \mathrm{E}-03$ & $5.12 \mathrm{E}+11$ & $7.81 \mathrm{E}-02$ & $3.91 \mathrm{E}+11$ & 7.74E-02 \\
\hline$(7,5)$ & $2.78 \mathrm{E}+11$ & $9.48 \mathrm{E}-02$ & $1.84 \mathrm{E}+12$ & $2.80 \mathrm{E}-01$ & $1.60 \mathrm{E}+12$ & $3.17 \mathrm{E}-01$ \\
\hline$(6,5)$ & $1.43 \mathrm{E}+12$ & 4.87E-01 & $1.89 \mathrm{E}+12$ & $2.89 \mathrm{E}-01$ & $1.34 \mathrm{E}+12$ & $2.65 \mathrm{E}-01$ \\
\hline$(7,3)$ & $2.15 \mathrm{E}+11$ & 7.33E-02 & $1.43 E+12$ & $2.17 \mathrm{E}-01$ & $1.17 \mathrm{E}+12$ & 2.32E-01 \\
\hline$(8,3)$ & $5.26 \mathrm{E}+10$ & $1.79 \mathrm{E}-02$ & $3.65 E+11$ & 5.57E-02 & $3.15 \mathrm{E}+11$ & $6.23 \mathrm{E}-02$ \\
\hline$(9,1)$ & $2.49 \mathrm{E}+10$ & 8.48E-03 & $1.81 \mathrm{E}+11$ & $2.76 \mathrm{E}-02$ & $2.04 \mathrm{E}+11$ & 4.05E-02 \\
\hline$(6,4)$ & $9.05 \mathrm{E}+11$ & 3.08E-01 & $3.45 E+11$ & $5.26 \mathrm{E}-02$ & $2.69 \mathrm{E}+10$ & 5.32E-03 \\
\hline
\end{tabular}




\begin{tabular}{|c|c|c|c|c|c|c|}
\hline \multirow[b]{2}{*}{ Chirality $(n, m)$} & \multicolumn{2}{|c|}{$88 \mathrm{mM}$ SDS - Ads } & \multicolumn{2}{|c|}{$88 \mathrm{mM}$ SDS - Elute } & \multicolumn{2}{|c|}{96 mM SDS - Control } \\
\hline & $\# S W N T / m L$ & fractional purity & $\# S W N T / m L$ & fractional purity & $\# S W N T / m L$ & fractional purity \\
\hline$(10,2)$ & $2.09 \mathrm{E}+08$ & $1.44 \mathrm{E}-04$ & $3.53 \mathrm{E}+10$ & $1.61 \mathrm{E}-02$ & $5.09 E+11$ & $6.86 \mathrm{E}-02$ \\
\hline$(7,5)$ & $1.98 \mathrm{E}+11$ & 1.37E-01 & $1.81 \mathrm{E}+11$ & 8.27E-02 & $1.85 \mathrm{E}+12$ & $2.49 \mathrm{E}-01$ \\
\hline$(6,5)$ & $5.25 \mathrm{E}+11$ & $3.63 \mathrm{E}-01$ & $1.03 \mathrm{E}+12$ & $4.70 \mathrm{E}-01$ & $1.93 E+12$ & $2.60 \mathrm{E}-01$ \\
\hline$(7,3)$ & $2.02 \mathrm{E}+11$ & $1.40 \mathrm{E}-01$ & $1.42 \mathrm{E}+11$ & $6.50 \mathrm{E}-02$ & $1.39 \mathrm{E}+12$ & $1.87 \mathrm{E}-01$ \\
\hline$(8,3)$ & $7.15 \mathrm{E}+10$ & 4.95E-02 & $3.40 \mathrm{E}+10$ & $1.55 \mathrm{E}-02$ & $4.64 \mathrm{E}+11$ & $6.26 \mathrm{E}-02$ \\
\hline$(9,1)$ & $3.72 \mathrm{E}+10$ & 2.57E-02 & $9.88 \mathrm{E}+09$ & $4.51 \mathrm{E}-03$ & $4.12 \mathrm{E}+11$ & $5.56 \mathrm{E}-02$ \\
\hline \multirow[t]{2}{*}{$(6,4)$} & $4.10 \mathrm{E}+11$ & 2.84E-01 & $7.58 \mathrm{E}+11$ & 3.46E-01 & $8.71 E+11$ & 1.17E-01 \\
\hline & \multicolumn{2}{|c|}{96 mM SDS - FT } & \multicolumn{2}{|c|}{96 mM SDS - Ads } & \multicolumn{2}{|c|}{$96 \mathrm{mM}$ SDS - Elute } \\
\hline Chirality $(n, m)$ & $\# S W N T / m L$ & fractional purity & \#SWNT/mL & fractional purity & \#SWNT/mL & fractional purity \\
\hline$(10,2)$ & $4.34 \mathrm{E}+11$ & 8.27E-02 & $1.03 \mathrm{E}+09$ & $8.10 \mathrm{E}-04$ & $4.99 \mathrm{E}+10$ & 3.07E-02 \\
\hline$(7,5)$ & $1.63 E+12$ & $3.11 \mathrm{E}-01$ & $1.91 \mathrm{E}+11$ & $1.51 \mathrm{E}-01$ & $1.46 \mathrm{E}+11$ & 9.01E-02 \\
\hline$(6,5)$ & $1.51 \mathrm{E}+12$ & $2.89 \mathrm{E}-01$ & $4.11 \mathrm{E}+11$ & $3.24 \mathrm{E}-01$ & $6.68 \mathrm{E}+11$ & 4.11E-01 \\
\hline$(7,3)$ & $1.14 \mathrm{E}+12$ & $2.17 \mathrm{E}-01$ & $1.91 \mathrm{E}+11$ & 1.50E-01 & $1.20 \mathrm{E}+11$ & 7.37E-02 \\
\hline$(8,3)$ & $2.82 \mathrm{E}+11$ & $5.38 \mathrm{E}-02$ & $6.85 \mathrm{E}+10$ & $5.40 \mathrm{E}-02$ & $3.07 \mathrm{E}+10$ & $1.89 \mathrm{E}-02$ \\
\hline$(9,1)$ & $1.75 \mathrm{E}+11$ & 3.33E-02 & $3.78 \mathrm{E}+10$ & 2.97E-02 & $4.67 \mathrm{E}+09$ & 2.87E-03 \\
\hline \multirow[t]{2}{*}{$(6,4)$} & $7.05 E+10$ & 1.35E-02 & $3.69 \mathrm{E}+11$ & 2.91E-01 & $6.06 \mathrm{E}+11$ & 3.73E-01 \\
\hline & \multicolumn{2}{|c|}{105 mM SDS - Control } & \multicolumn{2}{|c|}{105 mM SDS - FT } & \multicolumn{2}{|c|}{105 mM SDS - Ads } \\
\hline 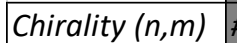 & \#SWNT/mL & fractional purity & $\# S W N T / m L$ & fractional purity & \#SWNT/mL & fractional purity \\
\hline$(10,2)$ & $4.96 \mathrm{E}+11$ & $6.92 \mathrm{E}-02$ & $4.39 \mathrm{E}+11$ & 8.34E-02 & $1.80 \mathrm{E}+10$ & 1.67E-02 \\
\hline$(7,5)$ & $1.79 \mathrm{E}+12$ & $2.50 \mathrm{E}-01$ & $1.61 \mathrm{E}+12$ & 3.05E-01 & $1.64 \mathrm{E}+11$ & 1.52E-01 \\
\hline$(6,5)$ & $1.90 \mathrm{E}+12$ & $2.66 \mathrm{E}-01$ & $1.60 \mathrm{E}+12$ & 3.03E-01 & $3.27 \mathrm{E}+11$ & 3.03E-01 \\
\hline$(7,3)$ & $1.29 \mathrm{E}+12$ & $1.80 \mathrm{E}-01$ & $1.08 \mathrm{E}+12$ & $2.06 \mathrm{E}-01$ & $1.64 \mathrm{E}+11$ & $1.52 \mathrm{E}-01$ \\
\hline$(8,3)$ & $4.44 \mathrm{E}+11$ & $6.20 \mathrm{E}-02$ & $2.67 \mathrm{E}+11$ & 5.07E-02 & $6.68 \mathrm{E}+10$ & $6.20 \mathrm{E}-02$ \\
\hline$(9,1)$ & $3.92 \mathrm{E}+11$ & $5.48 \mathrm{E}-02$ & $1.65 \mathrm{E}+11$ & 3.13E-02 & $3.39 E+10$ & $3.14 \mathrm{E}-02$ \\
\hline \multirow[t]{2}{*}{$(6,4)$} & $8.42 E+11$ & $1.18 \mathrm{E}-01$ & $1.09 \mathrm{E}+11$ & 2.07E-02 & $3.04 \mathrm{E}+11$ & 2.82E-01 \\
\hline & \multicolumn{2}{|c|}{105 mM SDS - Elute } & & & & \\
\hline Chirality $(n, m)$ & ) \#SWNT/mL & fractional pu & urity & & & \\
\hline$(10,2)$ & $6.88 \mathrm{E}+$ & 5.55 & $5 \mathrm{E}-02$ & & & \\
\hline$(7,5)$ & $1.51 \mathrm{E}_{+}$ & 1.22 & $2 \mathrm{E}-01$ & & & \\
\hline$(6,5)$ & $3.92 \mathrm{E}+$ & 3.17 & $7 \mathrm{E}-01$ & & & \\
\hline$(7,3)$ & $1.35 \mathrm{E}_{+}$ & 1.09 & $9 \mathrm{E}-01$ & & & \\
\hline$(8,3)$ & $3.02 \mathrm{E}+$ & 2.44 & $4 \mathrm{E}-02$ & & & \\
\hline$(9,1)$ & $5.61 \mathrm{E}+$ & 4.53 & $3 \mathrm{E}-03$ & & & \\
\hline$(6,4)$ & $4.56 \mathrm{E}+$ & 3.68 & $3 \mathrm{E}-01$ & & & \\
\hline
\end{tabular}




\begin{tabular}{|c|c|c|c|c|c|c|}
\hline \multicolumn{7}{|l|}{ Figure 3} \\
\hline & \multicolumn{2}{|c|}{$\rho=1.7$ Control } & \multicolumn{2}{|c|}{$\rho=1.7 \mathrm{FT}$} & \multicolumn{2}{|c|}{$\rho=1.7$ Adsorbed } \\
\hline Chirality $(n, m)$ & \#SWNT/mL & fractional purity & \#SWNT $/ \mathrm{mL}$ & fractional purity & \#SWNT/mL & fractional purity \\
\hline$(10,2)$ & $7.16 \mathrm{E}+11$ & $8.90 \mathrm{E}-02$ & $5.87 \mathrm{E}+11$ & $8.25 \mathrm{E}-02$ & $1.35 \mathrm{E}+09$ & $1.02 \mathrm{E}-03$ \\
\hline$(7,5)$ & $2.68 \mathrm{E}+12$ & $3.33 \mathrm{E}-01$ & $2.45 \mathrm{E}+12$ & $3.44 \mathrm{E}-01$ & $2.33 \mathrm{E}+11$ & $1.76 \mathrm{E}-01$ \\
\hline$(6,5)$ & $2.64 \mathrm{E}+12$ & $3.28 \mathrm{E}-01$ & $1.76 \mathrm{E}+12$ & $2.48 \mathrm{E}-01$ & $7.87 \mathrm{E}+11$ & $5.94 \mathrm{E}-01$ \\
\hline$(7,3)$ & $1.58 \mathrm{E}+12$ & $1.96 \mathrm{E}-01$ & $1.55 \mathrm{E}+12$ & $2.18 \mathrm{E}-01$ & $6.82 \mathrm{E}+10$ & $5.14 \mathrm{E}-02$ \\
\hline$(8,3)$ & $1.72 \mathrm{E}+11$ & $2.14 \mathrm{E}-02$ & $3.78 \mathrm{E}+11$ & $5.31 \mathrm{E}-02$ & $7.14 \mathrm{E}+09$ & 5.39E-03 \\
\hline$(9,1)$ & $2.63 E+11$ & $3.28 \mathrm{E}-02$ & $3.89 E+11$ & $5.46 \mathrm{E}-02$ & $5.02 \mathrm{E}+10$ & $3.78 \mathrm{E}-02$ \\
\hline \multirow[t]{2}{*}{$(6,4)$} & $1.02 E+08$ & 1.27E-05 & $2.76 \mathrm{E}+08$ & $3.88 \mathrm{E}-05$ & $1.78 \mathrm{E}+11$ & $1.35 \mathrm{E}-01$ \\
\hline & \multicolumn{2}{|c|}{$\rho=1.7$ Elute } & \multicolumn{2}{|c|}{$\rho=2.3$ Control } & \multicolumn{2}{|c|}{$\rho=2.3 \mathrm{FT}$} \\
\hline Chirality $(n, m)$ & \#SWNT/mL & fractional purity & \#SWNT/mL & fractional purity & \#SWNT/mL & fractional purity \\
\hline$(10,2)$ & $2.68 \mathrm{E}+10$ & $1.50 \mathrm{E}-02$ & $6.54 \mathrm{E}+11$ & $9.45 \mathrm{E}-02$ & $5.01 \mathrm{E}+11$ & $8.57 \mathrm{E}-02$ \\
\hline$(7,5)$ & $3.19 \mathrm{E}+11$ & $1.78 \mathrm{E}-01$ & $2.26 \mathrm{E}+12$ & $3.27 \mathrm{E}-01$ & $2.03 E+12$ & $3.47 \mathrm{E}-01$ \\
\hline$(6,5)$ & $1.15 \mathrm{E}+12$ & $6.40 \mathrm{E}-01$ & $2.14 \mathrm{E}+12$ & 3.09E-01 & $1.30 \mathrm{E}+12$ & $2.23 \mathrm{E}-01$ \\
\hline$(7,3)$ & $3.03 E+10$ & $1.69 \mathrm{E}-02$ & $1.44 \mathrm{E}+12$ & $2.09 \mathrm{E}-01$ & $1.30 \mathrm{E}+12$ & $2.23 \mathrm{E}-01$ \\
\hline$(8,3)$ & $1.19 \mathrm{E}+10$ & $6.65 \mathrm{E}-03$ & $1.91 \mathrm{E}+11$ & $2.76 \mathrm{E}-02$ & $3.62 \mathrm{E}+11$ & 6.19E-02 \\
\hline$(9,1)$ & $5.88 \mathrm{E}+10$ & $3.28 \mathrm{E}-02$ & $2.32 \mathrm{E}+11$ & $3.35 \mathrm{E}-02$ & $3.46 \mathrm{E}+11$ & $5.92 \mathrm{E}-02$ \\
\hline \multirow[t]{2}{*}{$(6,4)$} & $1.98 \mathrm{E}+11$ & $1.10 \mathrm{E}-01$ & $1.07 \mathrm{E}+08$ & $1.55 \mathrm{E}-05$ & $2.41 \mathrm{E}+08$ & $4.12 \mathrm{E}-05$ \\
\hline & \multicolumn{2}{|c|}{$\rho=2.3$ Adsorbed } & \multicolumn{2}{|c|}{$\rho=2.3$ Elute } & \multicolumn{2}{|c|}{$\rho=3.0$ Control } \\
\hline Chirality $(n, m)$ & $\# S W N T / m L$ & fractional purity & \#SWNT/mL & fractional purity & \#SWNT/mL & fractional purity \\
\hline$(10,2)$ & $4.16 \mathrm{E}+10$ & $2.87 \mathrm{E}-02$ & $2.41 \mathrm{E}+10$ & $1.22 \mathrm{E}-02$ & $4.95 \mathrm{E}+11$ & $9.08 \mathrm{E}-02$ \\
\hline$(7,5)$ & $2.39 E+11$ & $1.65 \mathrm{E}-01$ & $3.57 E+11$ & $1.81 \mathrm{E}-01$ & $1.79 \mathrm{E}+12$ & $3.28 \mathrm{E}-01$ \\
\hline$(6,5)$ & $7.45 \mathrm{E}+11$ & $5.15 \mathrm{E}-01$ & $1.27 \mathrm{E}+12$ & $6.46 \mathrm{E}-01$ & $1.70 \mathrm{E}+12$ & $3.11 \mathrm{E}-01$ \\
\hline$(7,3)$ & $1.96 \mathrm{E}+11$ & $1.36 \mathrm{E}-01$ & $3.35 \mathrm{E}+10$ & $1.70 \mathrm{E}-02$ & $1.11 \mathrm{E}+12$ & $2.04 \mathrm{E}-01$ \\
\hline$(8,3)$ & $5.94 \mathrm{E}+09$ & $4.11 \mathrm{E}-03$ & $6.50 \mathrm{E}+09$ & $3.30 \mathrm{E}-03$ & $1.70 \mathrm{E}+11$ & $3.12 \mathrm{E}-02$ \\
\hline$(9,1)$ & $4.68 \mathrm{E}+10$ & $3.24 \mathrm{E}-02$ & $6.49 \mathrm{E}+10$ & $3.29 \mathrm{E}-02$ & $1.92 \mathrm{E}+11$ & $3.52 \mathrm{E}-02$ \\
\hline \multirow[t]{2}{*}{$(6,4)$} & $1.72 \mathrm{E}+11$ & $1.19 \mathrm{E}-01$ & $2.11 \mathrm{E}+11$ & $1.07 \mathrm{E}-01$ & $9.83 \mathrm{E}+07$ & $1.80 \mathrm{E}-05$ \\
\hline & \multicolumn{2}{|c|}{$\rho=3.0 \mathrm{FT}$} & \multicolumn{2}{|c|}{$\rho=3.0$ Adsorbed } & \multicolumn{2}{|c|}{$\rho=3.0$ Elute } \\
\hline Chirality $(n, m)$ & \#SWNT/mL & fractional purity & \#SWNT/mL & fractional purity & \#SWNT/mL & fractional purity \\
\hline$(10,2)$ & $3.70 E+11$ & $8.76 \mathrm{E}-02$ & $1.41 \mathrm{E}+09$ & $9.68 \mathrm{E}-04$ & $8.17 E+07$ & $4.02 \mathrm{E}-05$ \\
\hline$(7,5)$ & $1.48 \mathrm{E}+12$ & $3.52 \mathrm{E}-01$ & $2.92 \mathrm{E}+11$ & $2.00 \mathrm{E}-01$ & $3.33 \mathrm{E}+11$ & 1.64E-01 \\
\hline$(6,5)$ & $8.61 \mathrm{E}+11$ & $2.04 \mathrm{E}-01$ & $7.87 \mathrm{E}+11$ & $5.40 \mathrm{E}-01$ & $1.35 \mathrm{E}+12$ & $6.62 \mathrm{E}-01$ \\
\hline$(7,3)$ & $9.87 E+11$ & $2.34 \mathrm{E}-01$ & $9.51 \mathrm{E}+10$ & $6.52 \mathrm{E}-02$ & $1.21 \mathrm{E}+10$ & 5.95E-03 \\
\hline$(8,3)$ & $2.56 \mathrm{E}+11$ & $6.06 \mathrm{E}-02$ & $4.37 \mathrm{E}+10$ & $3.00 \mathrm{E}-02$ & $4.70 \mathrm{E}+10$ & $2.31 \mathrm{E}-02$ \\
\hline$(9,1)$ & $2.63 E+11$ & $6.23 \mathrm{E}-02$ & $6.70 \mathrm{E}+10$ & $4.59 \mathrm{E}-02$ & $6.87 \mathrm{E}+10$ & $3.38 \mathrm{E}-02$ \\
\hline \multirow[t]{2}{*}{$(6,4)$} & $1.70 \mathrm{E}+08$ & 4.03E-05 & $1.72 \mathrm{E}+11$ & $1.18 \mathrm{E}-01$ & $2.26 \mathrm{E}+11$ & $1.11 \mathrm{E}-01$ \\
\hline & \multicolumn{2}{|c|}{$\rho=3.8$ Control } & \multicolumn{2}{|c|}{$\rho=3.8 \mathrm{FT}$} & \multicolumn{2}{|c|}{$\rho=3.8$ Adsorbed } \\
\hline Chirality $(n, m)$ & \#SWNT/mL & fractional purity & \#SWNT/mL & fractional purity & \#SWNT/mL & fractional purity \\
\hline$(10,2)$ & $3.89 \mathrm{E}+11$ & $9.36 \mathrm{E}-02$ & $2.79 \mathrm{E}+11$ & $9.28 \mathrm{E}-02$ & $4.43 \mathrm{E}+09$ & $3.31 \mathrm{E}-03$ \\
\hline$(7,5)$ & $1.36 \mathrm{E}+12$ & $3.26 \mathrm{E}-01$ & $1.08 \mathrm{E}+12$ & $3.60 \mathrm{E}-01$ & $2.64 \mathrm{E}+11$ & $1.98 \mathrm{E}-01$ \\
\hline$(6,5)$ & $1.29 \mathrm{E}+12$ & 3.09E-01 & $4.93 \mathrm{E}+11$ & $1.64 \mathrm{E}-01$ & $7.60 \mathrm{E}+11$ & 5.69E-01 \\
\hline$(7,3)$ & $8.51 \mathrm{E}+11$ & $2.05 \mathrm{E}-01$ & $7.44 \mathrm{E}+11$ & $2.48 \mathrm{E}-01$ & $7.17 \mathrm{E}+10$ & $5.37 \mathrm{E}-02$ \\
\hline$(8,3)$ & $1.33 \mathrm{E}+11$ & $3.19 \mathrm{E}-02$ & $2.09 \mathrm{E}+11$ & $6.94 \mathrm{E}-02$ & $2.73 \mathrm{E}+10$ & $2.04 \mathrm{E}-02$ \\
\hline$(9,1)$ & $1.45 \mathrm{E}+11$ & $3.49 \mathrm{E}-02$ & $2.00 E+11$ & $6.65 \mathrm{E}-02$ & $5.70 \mathrm{E}+10$ & $4.27 \mathrm{E}-02$ \\
\hline$(6,4)$ & $7.05 E+07$ & 1.69E-05 & $7.38 \mathrm{E}+07$ & 2.45E-05 & $1.51 \mathrm{E}+11$ & 1.13E-01 \\
\hline
\end{tabular}




\begin{tabular}{|l|r|r|r|r|r|r|}
\hline \multicolumn{2}{|c|}{} & \multicolumn{2}{|c|}{$\boldsymbol{\rho}=\mathbf{3 . 8}$ Elute } & \multicolumn{2}{c|}{$\boldsymbol{\rho}=\mathbf{4 . 5}$ Control } & \multicolumn{2}{c|}{$\boldsymbol{\rho}=\mathbf{4 . 5} \mathbf{F T}$} \\
\hline Chirality $(n, m)$ & $\# S W N T / m L$ & fractional purity & $\# S W N T / m L$ & fractional purity & $\# S W N T / m L$ & fractional purity \\
\hline $\mathbf{( 1 0 , 2 )}$ & $1.09 \mathrm{E}+08$ & $5.30 \mathrm{E}-05$ & $2.85 \mathrm{E}+11$ & $9.41 \mathrm{E}-02$ & $2.16 \mathrm{E}+11$ & $1.03 \mathrm{E}-01$ \\
\hline $\mathbf{( 7 , 5 )}$ & $3.57 \mathrm{E}+11$ & $1.74 \mathrm{E}-01$ & $9.87 \mathrm{E}+11$ & $3.25 \mathrm{E}-01$ & $7.69 \mathrm{E}+11$ & $3.67 \mathrm{E}-01$ \\
\hline $\mathbf{( 6 , 5 )}$ & $1.35 \mathrm{E}+12$ & $6.57 \mathrm{E}-01$ & $9.36 \mathrm{E}+11$ & $3.09 \mathrm{E}-01$ & $2.51 \mathrm{E}+11$ & $1.20 \mathrm{E}-01$ \\
\hline $\mathbf{( 7 , 3 )}$ & $1.85 \mathrm{E}+10$ & $9.04 \mathrm{E}-03$ & $6.26 \mathrm{E}+11$ & $2.07 \mathrm{E}-01$ & $5.43 \mathrm{E}+11$ & $2.60 \mathrm{E}-01$ \\
\hline $\mathbf{( 8 , 3 )}$ & $4.72 \mathrm{E}+10$ & $2.30 \mathrm{E}-02$ & $9.30 \mathrm{E}+10$ & $3.07 \mathrm{E}-02$ & $1.65 \mathrm{E}+11$ & $7.88 \mathrm{E}-02$ \\
\hline $\mathbf{( 9 , 1 )}$ & $6.99 \mathrm{E}+10$ & $3.41 \mathrm{E}-02$ & $1.04 \mathrm{E}+11$ & $3.44 \mathrm{E}-02$ & $1.48 \mathrm{E}+11$ & $7.08 \mathrm{E}-02$ \\
\hline $\mathbf{( 6 , 4 )}$ & $2.09 \mathrm{E}+11$ & $1.02 \mathrm{E}-01$ & $4.76 \mathrm{E}+07$ & $1.57 \mathrm{E}-05$ & $3.16 \mathrm{E}+07$ & $1.51 \mathrm{E}-05$ \\
\hline
\end{tabular}

\begin{tabular}{|l|r|r|r|r|}
\cline { 2 - 5 } \multicolumn{1}{c|}{} & \multicolumn{2}{c|}{$\boldsymbol{\rho = 4 . 5}$ Adsorbed } & \multicolumn{2}{c|}{$\boldsymbol{\rho}=\mathbf{4 . 5}$ Elute } \\
\hline Chirality $(n, m)$ & $\# S W N T / m L$ & fractional purity & \#SWNT/mL & fractional purity \\
\hline $\mathbf{( 1 0 , 2 )}$ & $3.13 \mathrm{E}+08$ & $2.78 \mathrm{E}-04$ & $1.46 \mathrm{E}+08$ & $7.70 \mathrm{E}-05$ \\
\hline $\mathbf{( 7 , 5 )}$ & $2.11 \mathrm{E}+11$ & $1.88 \mathrm{E}-01$ & $3.50 \mathrm{E}+11$ & $1.85 \mathrm{E}-01$ \\
\hline $\mathbf{( 6 , 5 )}$ & $6.41 \mathrm{E}+11$ & $5.69 \mathrm{E}-01$ & $1.23 \mathrm{E}+12$ & $6.48 \mathrm{E}-01$ \\
\hline $\mathbf{( 7 , 3 )}$ & $8.43 \mathrm{E}+10$ & $7.49 \mathrm{E}-02$ & $3.16 \mathrm{E}+10$ & $1.67 \mathrm{E}-02$ \\
\hline $\mathbf{( 8 , 3 )}$ & $2.28 \mathrm{E}+10$ & $2.03 \mathrm{E}-02$ & $4.49 \mathrm{E}+10$ & $2.37 \mathrm{E}-02$ \\
\hline $\mathbf{( 9 , 1 )}$ & $4.44 \mathrm{E}+10$ & $3.95 \mathrm{E}-02$ & $6.48 \mathrm{E}+10$ & $3.42 \mathrm{E}-02$ \\
\hline $\mathbf{( 6 , 4 )}$ & $1.22 \mathrm{E}+11$ & $1.08 \mathrm{E}-01$ & $1.76 \mathrm{E}+11$ & $9.30 \mathrm{E}-02$ \\
\hline
\end{tabular}

Table S3: SWCNT concentration and relative abundance of each chiral species for data presented in Figure $\mathbf{3}$ in the main text. 


\begin{tabular}{|l|r|r|r|r|r|r|}
\hline Figure 4 & \multicolumn{2}{|c|}{ Control } & \multicolumn{2}{c|}{ FT } & \multicolumn{2}{c|}{ Adsorbed } \\
\hline Chirality $(n, m)$ & \#SWNT/mL & fractional purity & \#SWNT/mL & fractional purity & \#SWNT/mL & fractional purity \\
\hline$(10,2)$ & $1.31 \mathrm{E}+12$ & $1.10 \mathrm{E}-01$ & $1.41 \mathrm{E}+12$ & $2.57 \mathrm{E}-01$ & $6.93 \mathrm{E}+09$ & $8.92 \mathrm{E}-04$ \\
\hline$(7,5)$ & $3.70 \mathrm{E}+12$ & $3.12 \mathrm{E}-01$ & $1.67 \mathrm{E}+12$ & $3.04 \mathrm{E}-01$ & $1.94 \mathrm{E}+12$ & $2.50 \mathrm{E}-01$ \\
\hline$(6,5)$ & $4.02 \mathrm{E}+12$ & $3.39 \mathrm{E}-01$ & $8.89 \mathrm{E}+10$ & $1.62 \mathrm{E}-02$ & $3.74 \mathrm{E}+12$ & $4.82 \mathrm{E}-01$ \\
\hline$(7,3)$ & $1.59 \mathrm{E}+12$ & $1.34 \mathrm{E}-01$ & $1.54 \mathrm{E}+12$ & $2.81 \mathrm{E}-01$ & $3.67 \mathrm{E}+11$ & $4.73 \mathrm{E}-02$ \\
\hline$(8,3)$ & $3.86 \mathrm{E}+11$ & $3.26 \mathrm{E}-02$ & $4.63 \mathrm{E}+11$ & $8.44 \mathrm{E}-02$ & $2.57 \mathrm{E}+11$ & $3.30 \mathrm{E}-02$ \\
\hline$(9,1)$ & $3.46 \mathrm{E}+11$ & $2.92 \mathrm{E}-02$ & $3.15 \mathrm{E}+11$ & $5.74 \mathrm{E}-02$ & $3.02 \mathrm{E}+11$ & $3.89 \mathrm{E}-02$ \\
\hline$(6,4)$ & $5.19 \mathrm{E}+11$ & $4.37 \mathrm{E}-02$ & $2.68 \mathrm{E}+07$ & $4.89 \mathrm{E}-06$ & $1.15 \mathrm{E}+12$ & $1.48 \mathrm{E}-01$ \\
\hline
\end{tabular}

\begin{tabular}{|l|r|r|}
\cline { 2 - 3 } \multicolumn{1}{c|}{} & \multicolumn{2}{c|}{ Elute } \\
\hline Chirality $(n, m)$ & \#SWNT/mL & fractional purity \\
\hline$(10,2)$ & $1.05 \mathrm{E}+11$ & $1.69 \mathrm{E}-02$ \\
\hline$(7,5)$ & $1.55 \mathrm{E}+12$ & $2.50 \mathrm{E}-01$ \\
\hline$(6,5)$ & $3.07 \mathrm{E}+12$ & $4.94 \mathrm{E}-01$ \\
\hline$(7,3)$ & $3.20 \mathrm{E}+11$ & $5.15 \mathrm{E}-02$ \\
\hline$(8,3)$ & $2.12 \mathrm{E}+11$ & $3.42 \mathrm{E}-02$ \\
\hline$(9,1)$ & $2.12 \mathrm{E}+11$ & $3.42 \mathrm{E}-02$ \\
\hline$(6,4)$ & $7.41 \mathrm{E}+11$ & $1.19 \mathrm{E}-01$ \\
\hline
\end{tabular}

Table S4: SWCNT concentration and relative abundance of each chiral species for data presented in Figure $\mathbf{4}$ in the main text. 


\begin{tabular}{|c|c|c|c|c|c|c|}
\hline Figure 5 & \multicolumn{2}{|c|}{ Neat gel/SWNT control 1} & \multicolumn{2}{|c|}{ Neat gel/SWNT FT 1} & \multicolumn{2}{|c|}{ Neat gel/SWNT Ads 1} \\
\hline Chirality $(n, m)$ & $\# S W N T / m L$ & fractional purity & $\# S W N T / m L$ & fractional purity & $\# S W N T / m L$ & fractional purity \\
\hline$(10,2)$ & $4.11 \mathrm{E}+11$ & 8.49E-02 & $3.76 \mathrm{E}+11$ & $9.04 \mathrm{E}-02$ & $1.37 \mathrm{E}+08$ & 2.06E-04 \\
\hline$(7,5)$ & $1.35 E+12$ & 2.80E-01 & $1.29 \mathrm{E}+12$ & 3.09E-01 & $5.79 \mathrm{E}+10$ & 8.72E-02 \\
\hline$(6,5)$ & $1.35 \mathrm{E}+12$ & $2.80 \mathrm{E}-01$ & $1.08 \mathrm{E}+12$ & $2.60 \mathrm{E}-01$ & $2.57 \mathrm{E}+11$ & 3.87E-01 \\
\hline$(7,3)$ & $1.10 E+12$ & $2.28 \mathrm{E}-01$ & $9.54 \mathrm{E}+11$ & 2.29E-01 & $1.25 \mathrm{E}+11$ & 1.89E-01 \\
\hline$(8,3)$ & $2.49 E+11$ & $5.15 \mathrm{E}-02$ & $2.36 \mathrm{E}+11$ & 5.67E-02 & $1.08 \mathrm{E}+10$ & 1.63E-02 \\
\hline$(9,1)$ & $1.32 \mathrm{E}+11$ & 2.73E-02 & $1.42 \mathrm{E}+11$ & $3.40 \mathrm{E}-02$ & $7.32 \mathrm{E}+09$ & 1.10E-02 \\
\hline \multirow[t]{2}{*}{$(6,4)$} & $2.37 \mathrm{E}+11$ & $4.90 \mathrm{E}-02$ & $8.58 \mathrm{E}+10$ & $2.06 \mathrm{E}-02$ & $2.06 \mathrm{E}+11$ & 3.09E-01 \\
\hline & \multicolumn{2}{|c|}{ Neat gel/SWNT control 2} & \multicolumn{2}{|c|}{ Neat gel/SWNT FT 2} & \multicolumn{2}{|c|}{ Neat gel/SWNT Ads 2} \\
\hline Chirality $(n, m)$ & $\# S W N T / m L$ & fractional purity & $\# S W N T / m L$ & fractional purity & \#SWNT/mL & fractional purity \\
\hline$(10,2)$ & $4.11 \mathrm{E}+11$ & $8.49 \mathrm{E}-02$ & $3.71 \mathrm{E}+11$ & $8.98 \mathrm{E}-02$ & $5.24 \mathrm{E}+07$ & 7.57E-05 \\
\hline$(7,5)$ & $1.35 \mathrm{E}+12$ & $2.80 \mathrm{E}-01$ & $1.27 \mathrm{E}+12$ & 3.07E-01 & $6.12 \mathrm{E}+10$ & 8.84E-02 \\
\hline$(6,5)$ & $1.35 \mathrm{E}+12$ & 2.80E-01 & $1.05 E+12$ & $2.54 \mathrm{E}-01$ & $2.91 \mathrm{E}+11$ & $4.20 \mathrm{E}-01$ \\
\hline$(7,3)$ & $1.10 \mathrm{E}+12$ & $2.28 \mathrm{E}-01$ & $1.01 \mathrm{E}+12$ & $2.44 \mathrm{E}-01$ & $7.26 \mathrm{E}+10$ & $1.05 \mathrm{E}-01$ \\
\hline$(8,3)$ & $2.49 E+11$ & $5.15 \mathrm{E}-02$ & $2.20 \mathrm{E}+11$ & 5.32E-02 & $3.39 E+10$ & 4.90E-02 \\
\hline$(9,1)$ & $1.32 \mathrm{E}+11$ & $2.73 E-02$ & $1.48 \mathrm{E}+11$ & 3.60E-02 & $1.44 \mathrm{E}+10$ & $2.08 \mathrm{E}-02$ \\
\hline \multirow[t]{2}{*}{$(6,4)$} & $2.37 \mathrm{E}+11$ & $4.90 \mathrm{E}-02$ & $6.48 \mathrm{E}+10$ & 1.57E-02 & $2.19 \mathrm{E}+11$ & 3.17E-01 \\
\hline & \multicolumn{2}{|c|}{ Neat gel/SWNT control 3} & \multicolumn{2}{|c|}{ Neat gel/SWNT FT 3} & \multicolumn{2}{|c|}{ Neat gel/SWNT Ads 3} \\
\hline Chirality $(n, m)$ & $\# S W N T / m L$ & fractional purity & \#SWNT/mL & fractional purity & \#SWNT/mL & fractional purity \\
\hline$(10,2)$ & $4.11 \mathrm{E}+11$ & $8.49 \mathrm{E}-02$ & $3.57 \mathrm{E}+11$ & $8.62 \mathrm{E}-02$ & $1.48 \mathrm{E}+08$ & $2.24 \mathrm{E}-04$ \\
\hline$(7,5)$ & $1.35 \mathrm{E}+12$ & $2.80 \mathrm{E}-01$ & $1.28 \mathrm{E}+12$ & 3.08E-01 & $5.68 \mathrm{E}+10$ & $8.55 \mathrm{E}-02$ \\
\hline$(6,5)$ & $1.35 E+12$ & 2.80E-01 & $1.05 \mathrm{E}+12$ & $2.54 \mathrm{E}-01$ & $2.85 E+11$ & $4.29 \mathrm{E}-01$ \\
\hline$(7,3)$ & $1.10 \mathrm{E}+12$ & 2.28E-01 & $9.98 \mathrm{E}+11$ & $2.41 \mathrm{E}-01$ & $8.03 E+10$ & $1.21 \mathrm{E}-01$ \\
\hline$(8,3)$ & $2.49 E+11$ & $5.15 \mathrm{E}-02$ & $2.41 \mathrm{E}+11$ & $5.81 \mathrm{E}-02$ & $1.89 \mathrm{E}+10$ & $2.84 \mathrm{E}-02$ \\
\hline$(9,1)$ & $1.32 \mathrm{E}+11$ & $2.73 \mathrm{E}-02$ & $1.53 \mathrm{E}+11$ & $3.70 \mathrm{E}-02$ & $9.93 \mathrm{E}+09$ & $1.50 \mathrm{E}-02$ \\
\hline \multirow[t]{2}{*}{$(6,4)$} & $2.37 \mathrm{E}+11$ & $4.90 \mathrm{E}-02$ & $6.69 \mathrm{E}+10$ & $1.61 \mathrm{E}-02$ & $2.13 \mathrm{E}+11$ & $3.21 \mathrm{E}-01$ \\
\hline & \multicolumn{2}{|c|}{ Neat gel/SWNT Elute 1} & \multicolumn{2}{|c|}{ Neat gel/Reclaimed SWNT Control 1} & \multicolumn{2}{|c|}{ Neat gel/Reclaimed SWNT FT 1} \\
\hline Chirality $(n, m)$ & $\# S W N T / m L$ & fractional purity & $\# S W N T / m L$ & fractional purity & $\# S W N T / m L$ & fractional purity \\
\hline$(10,2)$ & $2.73 \mathrm{E}+08$ & $8.57 \mathrm{E}-04$ & $8.17 \mathrm{E}+08$ & 1.92E-04 & $2.64 \mathrm{E}+08$ & 9.62E-05 \\
\hline$(7,5)$ & $2.93 \mathrm{E}+10$ & $9.20 \mathrm{E}-02$ & $1.61 \mathrm{E}+11$ & $3.78 \mathrm{E}-02$ & $1.67 \mathrm{E}+11$ & $6.10 \mathrm{E}-02$ \\
\hline$(6,5)$ & $1.59 \mathrm{E}+11$ & $5.00 \mathrm{E}-01$ & $2.55 \mathrm{E}+12$ & 5.97E-01 & $1.60 \mathrm{E}+12$ & $5.84 \mathrm{E}-01$ \\
\hline$(7,3)$ & $2.00 \mathrm{E}+10$ & $6.28 \mathrm{E}-02$ & $6.87 \mathrm{E}+11$ & $1.61 \mathrm{E}-01$ & $4.79 \mathrm{E}+11$ & $1.75 \mathrm{E}-01$ \\
\hline$(8,3)$ & $8.58 \mathrm{E}+09$ & $2.70 \mathrm{E}-02$ & $6.61 \mathrm{E}+10$ & $1.55 \mathrm{E}-02$ & $5.80 \mathrm{E}+10$ & $2.12 \mathrm{E}-02$ \\
\hline$(9,1)$ & $3.06 \mathrm{E}+09$ & $9.61 \mathrm{E}-03$ & $1.27 \mathrm{E}+11$ & $2.98 \mathrm{E}-02$ & $9.21 \mathrm{E}+10$ & 3.36E-02 \\
\hline \multirow[t]{2}{*}{$(6,4)$} & $9.81 \mathrm{E}+10$ & $3.08 \mathrm{E}-01$ & $6.78 \mathrm{E}+11$ & $1.59 \mathrm{E}-01$ & $3.46 \mathrm{E}+11$ & $1.26 \mathrm{E}-01$ \\
\hline & \multicolumn{2}{|c|}{ Neat gel/SWNT Elute 2} & \multicolumn{2}{|c|}{ Neat gel/Reclaimed SWNT Control 2} & \multicolumn{2}{|c|}{ Neat gel/Reclaimed SWNT FT 2} \\
\hline Chirality $(n, m)$ & $\# S W N T / m L$ & fractional purity & $\# S W N T / m L$ & fractional purity & $\# S W N T / m L$ & fractional purity \\
\hline$(10,2)$ & $9.78 \mathrm{E}+08$ & $2.93 \mathrm{E}-03$ & $8.17 \mathrm{E}+08$ & 1.92E-04 & $2.51 \mathrm{E}+08$ & 9.27E-05 \\
\hline$(7,5)$ & $3.06 \mathrm{E}+10$ & 9.17E-02 & $1.61 \mathrm{E}+11$ & $3.78 \mathrm{E}-02$ & $1.71 \mathrm{E}+11$ & 6.32E-02 \\
\hline$(6,5)$ & $1.66 \mathrm{E}+11$ & $4.98 \mathrm{E}-01$ & $2.55 E+12$ & 5.97E-01 & $1.56 \mathrm{E}+12$ & 5.77E-01 \\
\hline$(7,3)$ & $2.24 \mathrm{E}+10$ & $6.70 \mathrm{E}-02$ & $6.87 \mathrm{E}+11$ & $1.61 \mathrm{E}-01$ & $4.86 \mathrm{E}+11$ & $1.79 \mathrm{E}-01$ \\
\hline$(8,3)$ & $6.83 \mathrm{E}+09$ & $2.04 \mathrm{E}-02$ & $6.61 \mathrm{E}+10$ & 1.55E-02 & $6.24 \mathrm{E}+10$ & 2.30E-02 \\
\hline$(9,1)$ & $2.45 \mathrm{E}+09$ & 7.34E-03 & $1.27 \mathrm{E}+11$ & $2.98 \mathrm{E}-02$ & $9.29 \mathrm{E}+10$ & 3.43E-02 \\
\hline$(6,4)$ & $1.05 \mathrm{E}+11$ & $3.13 \mathrm{E}-01$ & $6.78 \mathrm{E}+11$ & $1.59 \mathrm{E}-01$ & $3.34 \mathrm{E}+11$ & $1.23 \mathrm{E}-01$ \\
\hline
\end{tabular}




\begin{tabular}{|l|r|r|r|r|r|r|}
\hline & \multicolumn{2}{|c|}{ Neat gel/SWNT Elute 3 } & \multicolumn{2}{c|}{ Neat gel/Reclaimed SWNT Control 3 } & \multicolumn{2}{c|}{ Neat gel/Reclaimed SWNT FT 3 } \\
\hline Chirality $(n, m)$ & \#SWNT/mL & fractional purity & \#SWNT/mL & fractional purity & \#SWNT/mL & fractional purity \\
\hline $\mathbf{( 1 0 , 2 )}$ & $3.16 \mathrm{E}+08$ & $8.96 \mathrm{E}-04$ & $8.17 \mathrm{E}+08$ & $1.92 \mathrm{E}-04$ & $2.84 \mathrm{E}+08$ & $1.05 \mathrm{E}-04$ \\
\hline $\mathbf{( 7 , 5 )}$ & $3.49 \mathrm{E}+10$ & $9.88 \mathrm{E}-02$ & $1.61 \mathrm{E}+11$ & $3.78 \mathrm{E}-02$ & $1.71 \mathrm{E}+11$ & $6.31 \mathrm{E}-02$ \\
\hline $\mathbf{( 6 , 5 )}$ & $1.73 \mathrm{E}+11$ & $4.90 \mathrm{E}-01$ & $2.55 \mathrm{E}+12$ & $5.97 \mathrm{E}-01$ & $1.58 \mathrm{E}+12$ & $5.83 \mathrm{E}-01$ \\
\hline $\mathbf{( 7 , 3 )}$ & $2.55 \mathrm{E}+10$ & $7.23 \mathrm{E}-02$ & $6.87 \mathrm{E}+11$ & $1.61 \mathrm{E}-01$ & $4.73 \mathrm{E}+11$ & $1.75 \mathrm{E}-01$ \\
\hline $\mathbf{( 8 , 3 )}$ & $8.94 \mathrm{E}+09$ & $2.53 \mathrm{E}-02$ & $6.61 \mathrm{E}+10$ & $1.55 \mathrm{E}-02$ & $6.00 \mathrm{E}+10$ & $2.22 \mathrm{E}-02$ \\
\hline $\mathbf{( 9 , 1 )}$ & $3.80 \mathrm{E}+09$ & $1.08 \mathrm{E}-02$ & $1.27 \mathrm{E}+11$ & $2.98 \mathrm{E}-02$ & $9.24 \mathrm{E}+10$ & $3.42 \mathrm{E}-02$ \\
\hline $\mathbf{( 6 , 4 )}$ & $1.07 \mathrm{E}+11$ & $3.02 \mathrm{E}-01$ & $6.78 \mathrm{E}+11$ & $1.59 \mathrm{E}-01$ & $3.32 \mathrm{E}+11$ & $1.23 \mathrm{E}-01$ \\
\hline
\end{tabular}

\begin{tabular}{|l|r|r|r|r|r|r|}
\hline & \multicolumn{2}{|c|}{ Neat gel/Reclaimed SWNT Ads 1 } & \multicolumn{2}{c|}{ Neat gel/Reclaimed SWNT Elute 1 } & \multicolumn{2}{c|}{ Reclaimed gel Neat SWNT Control 1 } \\
\hline Chirality $(n, m)$ & $\# S W N T / m L$ & fractional purity & \#SWNT/mL & fractional purity & \#SWNT/mL & fractional purity \\
\hline $\mathbf{( 1 0 , 2 )}$ & $1.78 \mathrm{E}+10$ & $1.14 \mathrm{E}-02$ & $2.88 \mathrm{E}+10$ & $2.73 \mathrm{E}-02$ & $4.11 \mathrm{E}+11$ & $8.49 \mathrm{E}-02$ \\
\hline $\mathbf{( 7 , 5 )}$ & $4.91 \mathrm{E}+07$ & $3.15 \mathrm{E}-05$ & $2.04 \mathrm{E}+10$ & $1.93 \mathrm{E}-02$ & $1.35 \mathrm{E}+12$ & $2.80 \mathrm{E}-01$ \\
\hline $\mathbf{( 6 , 5 )}$ & $9.51 \mathrm{E}+11$ & $6.11 \mathrm{E}-01$ & $7.15 \mathrm{E}+11$ & $6.77 \mathrm{E}-01$ & $1.35 \mathrm{E}+12$ & $2.80 \mathrm{E}-01$ \\
\hline $\mathbf{( 7 , 3 )}$ & $2.09 \mathrm{E}+11$ & $1.34 \mathrm{E}-01$ & $4.05 \mathrm{E}+10$ & $3.83 \mathrm{E}-02$ & $1.10 \mathrm{E}+12$ & $2.28 \mathrm{E}-01$ \\
\hline $\mathbf{( 8 , 3 )}$ & $9.81 \mathrm{E}+09$ & $6.30 \mathrm{E}-03$ & $1.88 \mathrm{E}+07$ & $1.78 \mathrm{E}-05$ & $2.49 \mathrm{E}+11$ & $5.15 \mathrm{E}-02$ \\
\hline $\mathbf{( 9 , 1 )}$ & $3.80 \mathrm{E}+10$ & $2.44 \mathrm{E}-02$ & $2.09 \mathrm{E}+10$ & $1.98 \mathrm{E}-02$ & $1.32 \mathrm{E}+11$ & $2.73 \mathrm{E}-02$ \\
\hline $\mathbf{( 6 , 4 )}$ & $3.32 \mathrm{E}+11$ & $2.13 \mathrm{E}-01$ & $2.30 \mathrm{E}+11$ & $2.18 \mathrm{E}-01$ & $2.37 \mathrm{E}+11$ & $4.90 \mathrm{E}-02$ \\
\hline
\end{tabular}

\begin{tabular}{|l|r|r|r|r|r|r|}
\hline & \multicolumn{2}{|c|}{ Neat gel/Reclaimed SWNT Ads 2 } & \multicolumn{2}{c|}{ Neat gel/Reclaimed SWNT Elute 2 } & \multicolumn{2}{l|}{ Reclaimed gel Neat SWNT Control 2 } \\
\hline Chirality $(n, m)$ & \#SWNT/mL & fractional purity & \#SWNT/mL & fractional purity & \#SWNT/mL & fractional purity \\
\hline $\mathbf{( 1 0 , 2 )}$ & $2.29 \mathrm{E}+10$ & $1.43 \mathrm{E}-02$ & $3.06 \mathrm{E}+10$ & $2.73 \mathrm{E}-02$ & $4.11 \mathrm{E}+11$ & $8.49 \mathrm{E}-02$ \\
\hline $\mathbf{( 7 , 5 )}$ & $3.83 \mathrm{E}+07$ & $2.38 \mathrm{E}-05$ & $1.60 \mathrm{E}+10$ & $1.43 \mathrm{E}-02$ & $1.35 \mathrm{E}+12$ & $2.80 \mathrm{E}-01$ \\
\hline $\mathbf{( 6 , 5 )}$ & $9.89 \mathrm{E}+11$ & $6.16 \mathrm{E}-01$ & $7.74 \mathrm{E}+11$ & $6.92 \mathrm{E}-01$ & $1.35 \mathrm{E}+12$ & $2.80 \mathrm{E}-01$ \\
\hline $\mathbf{( 7 , 3 )}$ & $2.02 \mathrm{E}+11$ & $1.26 \mathrm{E}-01$ & $2.69 \mathrm{E}+10$ & $2.40 \mathrm{E}-02$ & $1.10 \mathrm{E}+12$ & $2.28 \mathrm{E}-01$ \\
\hline $\mathbf{( 8 , 3 )}$ & $7.99 \mathrm{E}+09$ & $4.98 \mathrm{E}-03$ & $1.69 \mathrm{E}+07$ & $1.51 \mathrm{E}-05$ & $2.49 \mathrm{E}+11$ & $5.15 \mathrm{E}-02$ \\
\hline $\mathbf{( 9 , 1 )}$ & $3.83 \mathrm{E}+10$ & $2.39 \mathrm{E}-02$ & $2.18 \mathrm{E}+10$ & $1.94 \mathrm{E}-02$ & $1.32 \mathrm{E}+11$ & $2.73 \mathrm{E}-02$ \\
\hline $\mathbf{( 6 , 4 )}$ & $3.44 \mathrm{E}+11$ & $2.15 \mathrm{E}-01$ & $2.49 \mathrm{E}+11$ & $2.23 \mathrm{E}-01$ & $2.37 \mathrm{E}+11$ & $4.90 \mathrm{E}-02$ \\
\hline
\end{tabular}

\begin{tabular}{|l|r|r|r|r|r|r|}
\hline & \multicolumn{2}{|c|}{ Neat gel/Reclaimed SWNT Ads 3 } & \multicolumn{2}{c|}{ Neat gel/Reclaimed SWNT Elute 3 } & \multicolumn{2}{l|}{ Reclaimed gel Neat SWNT Control 3 } \\
\hline Chirality $(n, m)$ & \#SWNT/mL & fractional purity & \#SWNT/mL & fractional purity & \#SWNT/mL & fractional purity \\
\hline $\mathbf{( 1 0 , 2 )}$ & $2.03 \mathrm{E}+10$ & $1.27 \mathrm{E}-02$ & $2.88 \mathrm{E}+10$ & $2.62 \mathrm{E}-02$ & $4.11 \mathrm{E}+11$ & $8.49 \mathrm{E}-02$ \\
\hline $\mathbf{( 7 , 5 )}$ & $3.68 \mathrm{E}+07$ & $2.29 \mathrm{E}-05$ & $1.67 \mathrm{E}+10$ & $1.52 \mathrm{E}-02$ & $1.35 \mathrm{E}+12$ & $2.80 \mathrm{E}-01$ \\
\hline $\mathbf{( 6 , 5 )}$ & $9.76 \mathrm{E}+11$ & $6.08 \mathrm{E}-01$ & $7.60 \mathrm{E}+11$ & $6.93 \mathrm{E}-01$ & $1.35 \mathrm{E}+12$ & $2.80 \mathrm{E}-01$ \\
\hline $\mathbf{( 7 , 3 )}$ & $2.14 \mathrm{E}+11$ & $1.33 \mathrm{E}-01$ & $2.59 \mathrm{E}+10$ & $2.36 \mathrm{E}-02$ & $1.10 \mathrm{E}+12$ & $2.28 \mathrm{E}-01$ \\
\hline $\mathbf{( 8 , 3 )}$ & $9.18 \mathrm{E}+09$ & $5.72 \mathrm{E}-03$ & $1.71 \mathrm{E}+07$ & $1.56 \mathrm{E}-05$ & $2.49 \mathrm{E}+11$ & $5.15 \mathrm{E}-02$ \\
\hline $\mathbf{( 9 , 1 )}$ & $3.85 \mathrm{E}+10$ & $2.40 \mathrm{E}-02$ & $2.15 \mathrm{E}+10$ & $1.96 \mathrm{E}-02$ & $1.32 \mathrm{E}+11$ & $2.73 \mathrm{E}-02$ \\
\hline $\mathbf{( 6 , 4 )}$ & $3.47 \mathrm{E}+11$ & $2.16 \mathrm{E}-01$ & $2.44 \mathrm{E}+11$ & $2.22 \mathrm{E}-01$ & $2.37 \mathrm{E}+11$ & $4.90 \mathrm{E}-02$ \\
\hline
\end{tabular}

\begin{tabular}{|l|r|r|r|r|r|r|}
\hline & \multicolumn{2}{|c|}{ Reclaimed gel Neat SWNT FT 1 } & \multicolumn{2}{c|}{ Reclaimed gel Neat SWNT Ads 1 } & \multicolumn{2}{c|}{ Reclaimed gel Neat SWNT Elute 1 } \\
\hline Chirality $(n, m)$ & \#SWNT/mL & fractional purity & \#SWNT/mL & \multicolumn{1}{l|}{ fractional purity } & \multicolumn{1}{|l}{ \#SWNT/mL } & fractional purity \\
\hline $\mathbf{( 1 0 , 2 )}$ & $3.07 \mathrm{E}+11$ & $7.79 \mathrm{E}-02$ & $1.85 \mathrm{E}+10$ & $2.32 \mathrm{E}-02$ & $8.06 \mathrm{E}+08$ & $1.87 \mathrm{E}-03$ \\
\hline $\mathbf{( 7 , 5 )}$ & $1.25 \mathrm{E}+12$ & $3.19 \mathrm{E}-01$ & $9.42 \mathrm{E}+10$ & $1.18 \mathrm{E}-01$ & $3.46 \mathrm{E}+10$ & $8.05 \mathrm{E}-02$ \\
\hline $\mathbf{( 6 , 5 )}$ & $1.11 \mathrm{E}+12$ & $2.81 \mathrm{E}-01$ & $2.23 \mathrm{E}+11$ & $2.80 \mathrm{E}-01$ & $2.09 \mathrm{E}+11$ & $4.86 \mathrm{E}-01$ \\
\hline $\mathbf{( 7 , 3 )}$ & $8.67 \mathrm{E}+11$ & $2.20 \mathrm{E}-01$ & $2.13 \mathrm{E}+11$ & $2.67 \mathrm{E}-01$ & $2.89 \mathrm{E}+10$ & $6.72 \mathrm{E}-02$ \\
\hline $\mathbf{( 8 , 3 )}$ & $2.19 \mathrm{E}+11$ & $5.57 \mathrm{E}-02$ & $3.12 \mathrm{E}+10$ & $3.92 \mathrm{E}-02$ & $7.62 \mathrm{E}+09$ & $1.77 \mathrm{E}-02$ \\
\hline $\mathbf{( 9 , 1 )}$ & $1.42 \mathrm{E}+11$ & $3.61 \mathrm{E}-02$ & $8.56 \mathrm{E}+09$ & $1.07 \mathrm{E}-02$ & $3.20 \mathrm{E}+09$ & $7.44 \mathrm{E}-03$ \\
\hline $\mathbf{( 6 , 4 )}$ & $4.01 \mathrm{E}+10$ & $1.02 \mathrm{E}-02$ & $2.08 \mathrm{E}+11$ & $2.61 \mathrm{E}-01$ & $1.46 \mathrm{E}+11$ & $3.39 \mathrm{E}-01$ \\
\hline
\end{tabular}




\begin{tabular}{|l|r|r|r|r|r|r|}
\hline & \multicolumn{2}{|c|}{ Reclaimed gel Neat SWNT FT 2 } & \multicolumn{2}{c|}{ Reclaimed gel Neat SWNT Ads 2 } & \multicolumn{2}{c|}{ Reclaimed gel Neat SWNT Elute 2 } \\
\hline Chirality $(n, m)$ & \#SWNT/mL & fractional purity & \#SWNT/mL & fractional purity & \#SWNT/mL & fractional purity \\
\hline $\mathbf{( 1 0 , 2 )}$ & $3.24 \mathrm{E}+11$ & $8.09 \mathrm{E}-02$ & $7.56 \mathrm{E}+09$ & $1.01 \mathrm{E}-02$ & $1.54 \mathrm{E}+09$ & $3.36 \mathrm{E}-03$ \\
\hline $\mathbf{( 7 , 5 )}$ & $1.27 \mathrm{E}+12$ & $3.17 \mathrm{E}-01$ & $6.60 \mathrm{E}+10$ & $8.81 \mathrm{E}-02$ & $3.85 \mathrm{E}+10$ & $8.38 \mathrm{E}-02$ \\
\hline $\mathbf{( 6 , 5 )}$ & $1.07 \mathrm{E}+12$ & $2.68 \mathrm{E}-01$ & $2.53 \mathrm{E}+11$ & $3.37 \mathrm{E}-01$ & $2.19 \mathrm{E}+11$ & $4.77 \mathrm{E}-01$ \\
\hline $\mathbf{( 7 , 3 )}$ & $9.31 \mathrm{E}+11$ & $2.33 \mathrm{E}-01$ & $1.45 \mathrm{E}+11$ & $1.94 \mathrm{E}-01$ & $3.39 \mathrm{E}+10$ & $7.37 \mathrm{E}-02$ \\
\hline $\mathbf{( 8 , 3 )}$ & $2.20 \mathrm{E}+11$ & $5.49 \mathrm{E}-02$ & $3.20 \mathrm{E}+10$ & $4.27 \mathrm{E}-02$ & $7.95 \mathrm{E}+09$ & $1.73 \mathrm{E}-02$ \\
\hline $\mathbf{( 9 , 1 )}$ & $1.46 \mathrm{E}+11$ & $3.66 \mathrm{E}-02$ & $1.30 \mathrm{E}+10$ & $1.73 \mathrm{E}-02$ & $3.51 \mathrm{E}+09$ & $7.64 \mathrm{E}-03$ \\
\hline $\mathbf{( 6 , 4 )}$ & $4.03 \mathrm{E}+10$ & $1.01 \mathrm{E}-02$ & $2.33 \mathrm{E}+11$ & $3.10 \mathrm{E}-01$ & $1.55 \mathrm{E}+11$ & $3.37 \mathrm{E}-01$ \\
\hline
\end{tabular}

\begin{tabular}{|l|r|r|r|r|r|r|}
\hline & \multicolumn{2}{|c|}{ Reclaimed gel Neat SWNT FT 3 } & \multicolumn{2}{c|}{ Reclaimed gel Neat SWNT Ads 3 } & \multicolumn{2}{c|}{ Reclaimed gel Neat SWNT Elute 3 } \\
\hline Chirality $(n, m)$ & $\# S W N T / m L$ & fractional purity & \#SWNT/mL & fractional purity & \#SWNT/mL & fractional purity \\
\hline $\mathbf{( 1 0 , 2 )}$ & $3.33 \mathrm{E}+11$ & $8.23 \mathrm{E}-02$ & $4.99 \mathrm{E}+09$ & $6.82 \mathrm{E}-03$ & $3.37 \mathrm{E}+08$ & $9.22 \mathrm{E}-04$ \\
\hline $\mathbf{( 7 , 5 )}$ & $1.27 \mathrm{E}+12$ & $3.14 \mathrm{E}-01$ & $6.32 \mathrm{E}+10$ & $8.63 \mathrm{E}-02$ & $2.61 \mathrm{E}+10$ & $7.14 \mathrm{E}-02$ \\
\hline $\mathbf{( 6 , 5 )}$ & $1.07 \mathrm{E}+12$ & $2.65 \mathrm{E}-01$ & $2.60 \mathrm{E}+11$ & $3.55 \mathrm{E}-01$ & $1.79 \mathrm{E}+11$ & $4.90 \mathrm{E}-01$ \\
\hline $\mathbf{( 7 , 3 )}$ & $9.45 \mathrm{E}+11$ & $2.34 \mathrm{E}-01$ & $1.33 \mathrm{E}+11$ & $1.81 \mathrm{E}-01$ & $2.50 \mathrm{E}+10$ & $6.86 \mathrm{E}-02$ \\
\hline $\mathbf{( 8 , 3 )}$ & $2.33 \mathrm{E}+11$ & $5.77 \mathrm{E}-02$ & $2.18 \mathrm{E}+10$ & $2.98 \mathrm{E}-02$ & $7.33 \mathrm{E}+09$ & $2.01 \mathrm{E}-02$ \\
\hline $\mathbf{( 9 , 1 )}$ & $1.53 \mathrm{E}+11$ & $3.79 \mathrm{E}-02$ & $8.68 \mathrm{E}+09$ & $1.19 \mathrm{E}-02$ & $2.21 \mathrm{E}+09$ & $6.06 \mathrm{E}-03$ \\
\hline $\mathbf{( 6 , 4 )}$ & $3.82 \mathrm{E}+10$ & $9.45 \mathrm{E}-03$ & $2.40 \mathrm{E}+11$ & $3.28 \mathrm{E}-01$ & $1.25 \mathrm{E}+11$ & $3.43 \mathrm{E}-01$ \\
\hline
\end{tabular}

\begin{tabular}{|l|r|r|r|r|r|r|}
\hline & \multicolumn{2}{|c|}{ Reclaimed SWNT \& Gel Control 1 } & \multicolumn{2}{c|}{ Reclaimed SWNT \& Gel FT 1 } & \multicolumn{2}{c|}{ Reclaimed SWNT \& Gel Ads 1 } \\
\hline Chirality $(n, m)$ & \#SWNT/mL & fractional purity & \#SWNT/mL & fractional purity & \#SWNT/mL & fractional purity \\
\hline $\mathbf{( 1 0 , 2 )}$ & $8.17 \mathrm{E}+08$ & $1.92 \mathrm{E}-04$ & $1.88 \mathrm{E}+08$ & $7.31 \mathrm{E}-05$ & $2.29 \mathrm{E}+10$ & $1.32 \mathrm{E}-02$ \\
\hline $\mathbf{( 7 , 5 )}$ & $1.61 \mathrm{E}+11$ & $3.78 \mathrm{E}-02$ & $1.72 \mathrm{E}+11$ & $6.70 \mathrm{E}-02$ & $5.01 \mathrm{E}+07$ & $2.88 \mathrm{E}-05$ \\
\hline $\mathbf{( 6 , 5 )}$ & $2.55 \mathrm{E}+12$ & $5.97 \mathrm{E}-01$ & $1.63 \mathrm{E}+12$ & $6.33 \mathrm{E}-01$ & $9.39 \mathrm{E}+11$ & $5.40 \mathrm{E}-01$ \\
\hline $\mathbf{( 7 , 3 )}$ & $6.87 \mathrm{E}+11$ & $1.61 \mathrm{E}-01$ & $3.49 \mathrm{E}+11$ & $1.36 \mathrm{E}-01$ & $3.29 \mathrm{E}+11$ & $1.89 \mathrm{E}-01$ \\
\hline $\mathbf{( 8 , 3 )}$ & $6.61 \mathrm{E}+10$ & $1.55 \mathrm{E}-02$ & $3.29 \mathrm{E}+10$ & $1.28 \mathrm{E}-02$ & $2.95 \mathrm{E}+10$ & $1.70 \mathrm{E}-02$ \\
\hline $\mathbf{( 9 , 1 )}$ & $1.27 \mathrm{E}+11$ & $2.98 \mathrm{E}-02$ & $8.54 \mathrm{E}+10$ & $3.32 \mathrm{E}-02$ & $4.57 \mathrm{E}+10$ & $2.63 \mathrm{E}-02$ \\
\hline $\mathbf{( 6 , 4 )}$ & $6.78 \mathrm{E}+11$ & $1.59 \mathrm{E}-01$ & $3.04 \mathrm{E}+11$ & $1.18 \mathrm{E}-01$ & $3.72 \mathrm{E}+11$ & $2.14 \mathrm{E}-01$ \\
\hline
\end{tabular}

\begin{tabular}{|l|r|r|r|r|r|r|}
\hline & \multicolumn{2}{|c|}{ Reclaimed SWNT \& Gel Control $\mathbf{2}$} & \multicolumn{2}{c|}{ Reclaimed SWNT \& Gel FT 2 } & \multicolumn{2}{c|}{ Reclaimed SWNT \& Gel Ads 2 } \\
\hline Chirality $(n, m)$ & \#SWNT/mL & fractional purity & \#SWNT/mL & fractional purity & \#SWNT/mL & fractional purity \\
\hline $\mathbf{( 1 0 , 2 )}$ & $8.17 \mathrm{E}+08$ & $1.92 \mathrm{E}-04$ & $2.23 \mathrm{E}+08$ & $8.17 \mathrm{E}-05$ & $2.87 \mathrm{E}+10$ & $1.80 \mathrm{E}-02$ \\
\hline $\mathbf{( 7 , 5 )}$ & $1.61 \mathrm{E}+11$ & $3.78 \mathrm{E}-02$ & $1.76 \mathrm{E}+11$ & $6.43 \mathrm{E}-02$ & $2.93 \mathrm{E}+07$ & $1.84 \mathrm{E}-05$ \\
\hline $\mathbf{( 6 , 5 )}$ & $2.55 \mathrm{E}+12$ & $5.97 \mathrm{E}-01$ & $1.66 \mathrm{E}+12$ & $6.06 \mathrm{E}-01$ & $9.02 \mathrm{E}+11$ & $5.65 \mathrm{E}-01$ \\
\hline $\mathbf{( 7 , 3 )}$ & $6.87 \mathrm{E}+11$ & $1.61 \mathrm{E}-01$ & $4.27 \mathrm{E}+11$ & $1.56 \mathrm{E}-01$ & $2.58 \mathrm{E}+11$ & $1.62 \mathrm{E}-01$ \\
\hline $\mathbf{( 8 , 3 )}$ & $6.61 \mathrm{E}+10$ & $1.55 \mathrm{E}-02$ & $4.76 \mathrm{E}+10$ & $1.74 \mathrm{E}-02$ & $2.19 \mathrm{E}+10$ & $1.37 \mathrm{E}-02$ \\
\hline $\mathbf{( 9 , 1 )}$ & $1.27 \mathrm{E}+11$ & $2.98 \mathrm{E}-02$ & $9.19 \mathrm{E}+10$ & $3.36 \mathrm{E}-02$ & $4.04 \mathrm{E}+10$ & $2.53 \mathrm{E}-02$ \\
\hline $\mathbf{( 6 , 4 )}$ & $6.78 \mathrm{E}+11$ & $1.59 \mathrm{E}-01$ & $3.33 \mathrm{E}+11$ & $1.22 \mathrm{E}-01$ & $3.44 \mathrm{E}+11$ & $2.15 \mathrm{E}-01$ \\
\hline
\end{tabular}

\begin{tabular}{|l|r|r|r|r|r|r|}
\hline & \multicolumn{2}{|c|}{ Reclaimed SWNT \& Gel Control 3 } & \multicolumn{2}{c|}{ Reclaimed SWNT \& Gel FT 3 } & \multicolumn{2}{c|}{ Reclaimed SWNT \& Gel Ads 3 } \\
\hline Chirality $(n, m)$ & \#SWNT/mL & fractional purity & \#SWNT/mL & fractional purity & \#SWNT/mL & fractional purity \\
\hline $\mathbf{( 1 0 , 2 )}$ & $8.17 \mathrm{E}+08$ & $1.92 \mathrm{E}-04$ & $2.23 \mathrm{E}+08$ & $8.09 \mathrm{E}-05$ & $2.83 \mathrm{E}+10$ & $1.81 \mathrm{E}-02$ \\
\hline $\mathbf{( 7 , 5 )}$ & $1.61 \mathrm{E}+11$ & $3.78 \mathrm{E}-02$ & $1.74 \mathrm{E}+11$ & $6.30 \mathrm{E}-02$ & $3.06 \mathrm{E}+07$ & $1.96 \mathrm{E}-05$ \\
\hline $\mathbf{( 6 , 5 )}$ & $2.55 \mathrm{E}+12$ & $5.97 \mathrm{E}-01$ & $1.66 \mathrm{E}+12$ & $6.02 \mathrm{E}-01$ & $8.93 \mathrm{E}+11$ & $5.72 \mathrm{E}-01$ \\
\hline $\mathbf{( 7 , 3 )}$ & $6.87 \mathrm{E}+11$ & $1.61 \mathrm{E}-01$ & $4.53 \mathrm{E}+11$ & $1.64 \mathrm{E}-01$ & $2.34 \mathrm{E}+11$ & $1.50 \mathrm{E}-01$ \\
\hline $\mathbf{( 8 , 3 )}$ & $6.61 \mathrm{E}+10$ & $1.55 \mathrm{E}-02$ & $4.96 \mathrm{E}+10$ & $1.80 \mathrm{E}-02$ & $2.03 \mathrm{E}+10$ & $1.30 \mathrm{E}-02$ \\
\hline $\mathbf{( 9 , 1 )}$ & $1.27 \mathrm{E}+11$ & $2.98 \mathrm{E}-02$ & $9.39 \mathrm{E}+10$ & $3.40 \mathrm{E}-02$ & $3.82 \mathrm{E}+10$ & $2.45 \mathrm{E}-02$ \\
\hline $\mathbf{( 6 , 4 )}$ & $6.78 \mathrm{E}+11$ & $1.59 \mathrm{E}-01$ & $3.29 \mathrm{E}+11$ & $1.19 \mathrm{E}-01$ & $3.47 \mathrm{E}+11$ & $2.23 \mathrm{E}-01$ \\
\hline
\end{tabular}




\begin{tabular}{|c|c|c|c|}
\hline & \multicolumn{2}{|c|}{ Reclaimed SWNT \& Gel Elute 3} & \\
\hline Chirality $(n, m)$ & \#SWNT/mL & fractional purity & \\
\hline$(10,2)$ & $3.40 \mathrm{E}+10$ & $2.68 \mathrm{E}-02$ & \\
\hline$(7,5)$ & $1.69 \mathrm{E}+10$ & 1.34E-02 & \\
\hline$(6,5)$ & $8.71 \mathrm{E}+11$ & 6.87E-01 & \\
\hline$(7,3)$ & $2.65 \mathrm{E}+10$ & $2.09 \mathrm{E}-02$ & \\
\hline$(8,3)$ & $1.59 \mathrm{E}+07$ & $1.25 \mathrm{E}-05$ & \\
\hline$(9,1)$ & $2.28 \mathrm{E}+10$ & $1.80 \mathrm{E}-02$ & \\
\hline \multirow[t]{2}{*}{$(6,4)$} & $2.96 \mathrm{E}+11$ & $2.34 \mathrm{E}-01$ & \\
\hline & \multicolumn{2}{|c|}{ Reclaimed SWNT \& Gel Elute 3} & \\
\hline Chirality $(n, m)$ & \#SWNT/mL & fractional purity & \\
\hline$(10,2)$ & $3.21 \mathrm{E}+10$ & $2.80 \mathrm{E}-02$ & \\
\hline$(7,5)$ & $1.50 \mathrm{E}+10$ & $1.31 \mathrm{E}-02$ & \\
\hline$(6,5)$ & $7.86 \mathrm{E}+11$ & $6.87 \mathrm{E}-01$ & \\
\hline$(7,3)$ & $2.17 \mathrm{E}+10$ & 1.90E-02 & \\
\hline$(8,3)$ & $1.70 \mathrm{E}+07$ & $1.48 \mathrm{E}-05$ & \\
\hline$(9,1)$ & $2.05 \mathrm{E}+10$ & 1.79E-02 & \\
\hline \multirow[t]{2}{*}{$(6,4)$} & $2.69 \mathrm{E}+11$ & $2.35 \mathrm{E}-01$ & \\
\hline & \multicolumn{2}{|c|}{ Reclaimed SWNT \& Gel Elute 3} & \\
\hline Chirality $(n, m)$ & \#SWNT/mL & fractional purity & \\
\hline$(10,2)$ & $2.98 \mathrm{E}+10$ & $2.56 \mathrm{E}-02$ & \\
\hline$(7,5)$ & $1.39 \mathrm{E}+10$ & $1.20 \mathrm{E}-02$ & \\
\hline$(6,5)$ & $8.01 \mathrm{E}+11$ & $6.90 \mathrm{E}-01$ & \\
\hline$(7,3)$ & $1.85 \mathrm{E}+10$ & $1.59 \mathrm{E}-02$ & \\
\hline$(8,3)$ & $1.73 \mathrm{E}+07$ & 1.49E-05 & \\
\hline$(9,1)$ & $1.99 \mathrm{E}+10$ & $1.71 \mathrm{E}-02$ & \\
\hline$(6,4)$ & $2.79 \mathrm{E}+11$ & $2.40 \mathrm{E}-01$ & \\
\hline
\end{tabular}




\begin{tabular}{|c|c|c|c|c|c|}
\hline Figure 6 & \multicolumn{5}{|c|}{$44 \mathrm{mM}$ SDS } \\
\hline & Run 1 & Run 2 & Run 3 & Ave & Stdev \\
\hline time (min) & \# SWNT eluted & \# SWNT eluted & \# SWNT eluted & \# SWNT eluted & \# SWNT eluted \\
\hline 0 & $3.06 \mathrm{E}+12$ & $3.06 \mathrm{E}+12$ & $3.06 \mathrm{E}+12$ & $3.06 \mathrm{E}+12$ & $0.00 \mathrm{E}+00$ \\
\hline 40 & $1.79 \mathrm{E}+12$ & $1.77 \mathrm{E}+12$ & $1.79 \mathrm{E}+12$ & $1.78 \mathrm{E}+12$ & $9.82 \mathrm{E}+09$ \\
\hline 61 & $1.79 \mathrm{E}+12$ & $1.92 \mathrm{E}+12$ & $1.80 \mathrm{E}+12$ & $1.84 \mathrm{E}+12$ & $7.41 \mathrm{E}+10$ \\
\hline 241 & $1.63 E+12$ & $1.55 \mathrm{E}+12$ & $1.58 \mathrm{E}+12$ & $1.59 \mathrm{E}+12$ & $4.28 \mathrm{E}+10$ \\
\hline 486 & $1.43 \mathrm{E}+12$ & $1.39 \mathrm{E}+12$ & $1.43 \mathrm{E}+12$ & $1.42 \mathrm{E}+12$ & $1.96 \mathrm{E}+10$ \\
\hline 1361 & $1.40 \mathrm{E}+12$ & $1.41 \mathrm{E}+12$ & $1.41 \mathrm{E}+12$ & $1.41 \mathrm{E}+12$ & $4.91 \mathrm{E}+09$ \\
\hline 3100 & $1.30 \mathrm{E}+12$ & $1.09 \mathrm{E}+12$ & $1.17 \mathrm{E}+12$ & $1.19 \mathrm{E}+12$ & $1.07 \mathrm{E}+11$ \\
\hline \multirow[t]{3}{*}{4422} & $1.16 \mathrm{E}+12$ & $1.19 \mathrm{E}+12$ & $1.19 \mathrm{E}+12$ & $1.18 \mathrm{E}+12$ & $1.96 \mathrm{E}+10$ \\
\hline & \multicolumn{5}{|c|}{$53 \mathrm{mM}$ SDS } \\
\hline & Run 1 & Run 2 & Run 3 & Ave & Stdev \\
\hline time $(\min )$ & \# SWNT eluted & \# SWNT eluted & \# SWNT eluted & \# SWNT eluted & \# SWNT eluted \\
\hline 0 & $2.66 \mathrm{E}+12$ & $2.66 \mathrm{E}+12$ & $2.66 \mathrm{E}+12$ & $2.66 \mathrm{E}+12$ & $0.00 \mathrm{E}+00$ \\
\hline 40 & $1.65 \mathrm{E}+12$ & $1.67 \mathrm{E}+12$ & $1.57 \mathrm{E}+12$ & $1.63 \mathrm{E}+12$ & $4.98 \mathrm{E}+10$ \\
\hline 61 & $1.79 \mathrm{E}+12$ & $1.70 \mathrm{E}+12$ & $1.58 \mathrm{E}+12$ & $1.69 \mathrm{E}+12$ & $1.02 \mathrm{E}+11$ \\
\hline 241 & $1.28 \mathrm{E}+12$ & $1.31 \mathrm{E}+12$ & $1.12 \mathrm{E}+12$ & $1.24 \mathrm{E}+12$ & $9.96 \mathrm{E}+10$ \\
\hline 486 & $1.23 \mathrm{E}+12$ & $1.19 \mathrm{E}+12$ & $1.24 \mathrm{E}+12$ & $1.22 \mathrm{E}+12$ & $2.73 \mathrm{E}+10$ \\
\hline 1361 & $4.85 \mathrm{E}+11$ & $9.86 \mathrm{E}+11$ & $1.09 \mathrm{E}+12$ & $8.53 E+11$ & $3.23 E+11$ \\
\hline 3100 & $9.95 \mathrm{E}+11$ & $8.08 \mathrm{E}+11$ & $9.35 E+11$ & $9.13 E+11$ & $9.56 \mathrm{E}+10$ \\
\hline \multirow[t]{3}{*}{4422} & $6.80 \mathrm{E}+11$ & $9.52 \mathrm{E}+11$ & $7.48 \mathrm{E}+11$ & $7.94 \mathrm{E}+11$ & $1.42 \mathrm{E}+11$ \\
\hline & \multicolumn{5}{|c|}{$62 \mathrm{mM}$ SDS } \\
\hline & Run 1 & Run 2 & Run 3 & Ave & Stdev \\
\hline time (min) & \# SWNT eluted & \# SWNT eluted & \# SWNT eluted & \# SWNT eluted & \# SWNT eluted \\
\hline 0 & $3.22 \mathrm{E}+12$ & $3.22 \mathrm{E}+12$ & $3.22 \mathrm{E}+12$ & $3.22 \mathrm{E}+12$ & $0.00 \mathrm{E}+00$ \\
\hline 40 & $1.55 \mathrm{E}+12$ & $1.73 \mathrm{E}+12$ & $1.82 \mathrm{E}+12$ & $1.70 \mathrm{E}+12$ & $1.39 \mathrm{E}+11$ \\
\hline 61 & $1.83 \mathrm{E}+12$ & $1.99 \mathrm{E}+12$ & $1.99 \mathrm{E}+12$ & $1.94 \mathrm{E}+12$ & $9.33 \mathrm{E}+10$ \\
\hline 241 & $1.50 \mathrm{E}+12$ & $1.53 \mathrm{E}+12$ & $1.45 \mathrm{E}+12$ & $1.49 \mathrm{E}+12$ & $3.83 \mathrm{E}+10$ \\
\hline 486 & $1.38 \mathrm{E}+12$ & $1.39 \mathrm{E}+12$ & $1.45 \mathrm{E}+12$ & $1.41 \mathrm{E}+12$ & $3.54 \mathrm{E}+10$ \\
\hline 1361 & $1.29 \mathrm{E}+12$ & $1.21 \mathrm{E}+12$ & $1.24 \mathrm{E}+12$ & $1.25 \mathrm{E}+12$ & $4.28 \mathrm{E}+10$ \\
\hline 3100 & $1.12 \mathrm{E}+12$ & $1.14 \mathrm{E}+12$ & $1.28 \mathrm{E}+12$ & $1.18 \mathrm{E}+12$ & $8.39 \mathrm{E}+10$ \\
\hline 4422 & $1.00 \mathrm{E}+12$ & $1.00 \mathrm{E}+12$ & $1.00 \mathrm{E}+12$ & $1.00 \mathrm{E}+12$ & $0.00 \mathrm{E}+00$ \\
\hline
\end{tabular}




\begin{tabular}{|c|c|c|c|c|c|}
\hline & \multicolumn{5}{|c|}{$70 \mathrm{mM}$ SDS } \\
\hline & Run 1 & Run 2 & Run 3 & Ave & Stdev \\
\hline time (min) & \# SWNT eluted & \# SWNT eluted & \# SWNT eluted & \# SWNT eluted & \# SWNT eluted \\
\hline 0 & $2.79 \mathrm{E}+12$ & $2.79 \mathrm{E}+12$ & $2.79 \mathrm{E}+12$ & $2.79 \mathrm{E}+12$ & $0.00 E+00$ \\
\hline 37 & $1.39 \mathrm{E}+12$ & $1.46 \mathrm{E}+12$ & $1.41 \mathrm{E}+12$ & $1.42 \mathrm{E}+12$ & $3.54 \mathrm{E}+10$ \\
\hline 66 & $1.45 \mathrm{E}+12$ & $1.41 \mathrm{E}+12$ & $1.50 \mathrm{E}+12$ & $1.45 \mathrm{E}+12$ & $4.28 \mathrm{E}+10$ \\
\hline 244 & $1.21 \mathrm{E}+12$ & $1.13 \mathrm{E}+12$ & $1.19 \mathrm{E}+12$ & $1.18 \mathrm{E}+12$ & $4.02 \mathrm{E}+10$ \\
\hline 500 & $1.12 \mathrm{E}+12$ & $1.07 \mathrm{E}+12$ & $9.86 \mathrm{E}+11$ & $1.06 \mathrm{E}+12$ & $6.87 \mathrm{E}+10$ \\
\hline 1448 & $9.35 \mathrm{E}+11$ & $9.52 \mathrm{E}+11$ & $9.52 \mathrm{E}+11$ & $9.47 \mathrm{E}+11$ & $9.82 E+09$ \\
\hline 2765 & $8.67 E+11$ & $8.08 \mathrm{E}+11$ & $8.33 \mathrm{E}+11$ & $8.36 \mathrm{E}+11$ & $2.99 E+10$ \\
\hline 4192 & $7.31 \mathrm{E}+11$ & $7.82 E+11$ & $7.23 \mathrm{E}+11$ & $7.45 E+11$ & $3.22 E+10$ \\
\hline
\end{tabular}

\begin{tabular}{|r|r|r|r|r|r|}
\hline & \multicolumn{7}{|c|}{79 mM SDS } \\
\hline & Run 1 & Run 2 & \multicolumn{1}{|l|}{ Run 3 } & Ave & Stdev \\
\hline time (min) \# SWNT eluted & \# SWNT eluted & \# SWNT eluted & \# SWNT eluted & \# SWNT eluted \\
\hline $\mathbf{0}$ & $3.03 \mathrm{E}+12$ & $3.03 \mathrm{E}+12$ & $3.03 \mathrm{E}+12$ & $3.03 \mathrm{E}+12$ & $0.00 \mathrm{E}+00$ \\
\hline $\mathbf{4 0}$ & $1.31 \mathrm{E}+12$ & $1.24 \mathrm{E}+12$ & $1.33 \mathrm{E}+12$ & $1.29 \mathrm{E}+12$ & $4.50 \mathrm{E}+10$ \\
\hline $\mathbf{6 1}$ & $1.23 \mathrm{E}+12$ & $1.43 \mathrm{E}+12$ & $1.29 \mathrm{E}+12$ & $1.32 \mathrm{E}+12$ & $1.00 \mathrm{E}+11$ \\
\hline $\mathbf{2 4 1}$ & $1.00 \mathrm{E}+12$ & $1.05 \mathrm{E}+12$ & $1.05 \mathrm{E}+12$ & $1.03 \mathrm{E}+12$ & $2.73 \mathrm{E}+10$ \\
\hline $\mathbf{4 8 6}$ & $9.01 \mathrm{E}+11$ & $9.10 \mathrm{E}+11$ & $9.35 \mathrm{E}+11$ & $9.15 \mathrm{E}+11$ & $1.77 \mathrm{E}+10$ \\
\hline $\mathbf{1 3 6 1}$ & $7.48 \mathrm{E}+11$ & $8.50 \mathrm{E}+11$ & $6.80 \mathrm{E}+11$ & $7.60 \mathrm{E}+11$ & $8.56 \mathrm{E}+10$ \\
\hline $\mathbf{3 1 0 0}$ & $6.46 \mathrm{E}+11$ & $6.80 \mathrm{E}+11$ & $7.82 \mathrm{E}+11$ & $7.03 \mathrm{E}+11$ & $7.08 \mathrm{E}+10$ \\
\hline $\mathbf{4 4 2 2}$ & $6.04 \mathrm{E}+11$ & $6.21 \mathrm{E}+11$ & $6.21 \mathrm{E}+11$ & $6.15 \mathrm{E}+11$ & $9.82 \mathrm{E}+09$ \\
\hline
\end{tabular}

\begin{tabular}{|r|r|r|r|r|r|}
\hline & \multicolumn{7}{|l|}{$\mathbf{8 8 ~ m M ~ S D S}$} \\
\hline & \multicolumn{7}{|l|}{ Run 1 } & Run 2 & Run 3 & Ave & Stdev \\
\hline time (min) & \# SWNT eluted & \# SWNT eluted & \# SWNT eluted & \# SWNT eluted & \# SWNT eluted \\
\hline $\mathbf{0}$ & $2.86 \mathrm{E}+12$ & $2.86 \mathrm{E}+12$ & $2.86 \mathrm{E}+12$ & $2.86 \mathrm{E}+12$ & $0.00 \mathrm{E}+00$ \\
\hline $\mathbf{3 7}$ & $8.84 \mathrm{E}+11$ & $9.10 \mathrm{E}+11$ & $8.76 \mathrm{E}+11$ & $8.90 \mathrm{E}+11$ & $1.77 \mathrm{E}+10$ \\
\hline $\mathbf{6 6}$ & $9.86 \mathrm{E}+11$ & $1.05 \mathrm{E}+12$ & $1.00 \mathrm{E}+12$ & $1.01 \mathrm{E}+12$ & $3.54 \mathrm{E}+10$ \\
\hline $\mathbf{2 4 4}$ & $7.91 \mathrm{E}+11$ & $7.48 \mathrm{E}+11$ & $7.82 \mathrm{E}+11$ & $7.74 \mathrm{E}+11$ & $2.25 \mathrm{E}+10$ \\
\hline $\mathbf{5 0 0}$ & $6.80 \mathrm{E}+11$ & $7.14 \mathrm{E}+11$ & $6.46 \mathrm{E}+11$ & $6.80 \mathrm{E}+11$ & $3.40 \mathrm{E}+10$ \\
\hline $\mathbf{1 4 4 8}$ & $5.53 \mathrm{E}+11$ & $5.78 \mathrm{E}+11$ & $5.44 \mathrm{E}+11$ & $5.58 \mathrm{E}+11$ & $1.77 \mathrm{E}+10$ \\
\hline $\mathbf{2 7 6 5}$ & $4.93 \mathrm{E}+11$ & $4.93 \mathrm{E}+11$ & $4.68 \mathrm{E}+11$ & $4.85 \mathrm{E}+11$ & $1.47 \mathrm{E}+10$ \\
\hline $\mathbf{4 1 9 2}$ & $4.34 \mathrm{E}+11$ & $3.74 \mathrm{E}+11$ & $4.42 \mathrm{E}+11$ & $4.17 \mathrm{E}+11$ & $3.71 \mathrm{E}+10$ \\
\hline
\end{tabular}

Table S6: Number of SWCNT eluted at specified time steps for data presented in Figure $\mathbf{5}$ of the main text. 


\begin{tabular}{|l|r|r|r|r|r|r|}
\hline Figure 7 & \multicolumn{2}{|c|}{ Control: } & \multicolumn{2}{c|}{ SDS, low - Ads } & \multicolumn{2}{c|}{ SDS, low - Elute } \\
\hline Chirality $(\mathbf{n}, \mathbf{m})$ & \#SWNT/mL & fractional purity & \#SWNT/mL & fractional purity & \#SWNT/mL & fractional purity \\
\hline $\mathbf{( 1 0 , 2 )}$ & $8.22 \mathrm{E}+11$ & $1.58 \mathrm{E}-01$ & $6.30 \mathrm{E}+09$ & $6.23 \mathrm{E}-03$ & $6.13 \mathrm{E}+09$ & $1.11 \mathrm{E}-02$ \\
\hline $\mathbf{( 7 , 5 )}$ & $1.40 \mathrm{E}+12$ & $2.69 \mathrm{E}-01$ & $6.83 \mathrm{E}+10$ & $6.75 \mathrm{E}-02$ & $5.43 \mathrm{E}+10$ & $9.84 \mathrm{E}-02$ \\
\hline $\mathbf{( 6 , 5 )}$ & $1.29 \mathrm{E}+12$ & $2.48 \mathrm{E}-01$ & $4.69 \mathrm{E}+11$ & $4.64 \mathrm{E}-01$ & $2.68 \mathrm{E}+11$ & $4.87 \mathrm{E}-01$ \\
\hline $\mathbf{( 7 , 3 )}$ & $1.31 \mathrm{E}+12$ & $2.52 \mathrm{E}-01$ & $1.13 \mathrm{E}+11$ & $1.12 \mathrm{E}-01$ & $4.01 \mathrm{E}+10$ & $7.28 \mathrm{E}-02$ \\
\hline $\mathbf{( 8 , 3 )}$ & $1.34 \mathrm{E}+11$ & $2.57 \mathrm{E}-02$ & $1.65 \mathrm{E}+07$ & $1.64 \mathrm{E}-05$ & $2.53 \mathrm{E}+09$ & $4.59 \mathrm{E}-03$ \\
\hline $\mathbf{( 9 , 1 )}$ & $2.29 \mathrm{E}+11$ & $4.39 \mathrm{E}-02$ & $4.35 \mathrm{E}+09$ & $4.30 \mathrm{E}-03$ & $7.97 \mathrm{E}+09$ & $1.45 \mathrm{E}-02$ \\
\hline $\mathbf{( 6 , 4 )}$ & $1.98 \mathrm{E}+10$ & $3.81 \mathrm{E}-03$ & $3.50 \mathrm{E}+11$ & $3.46 \mathrm{E}-01$ & $1.72 \mathrm{E}+11$ & $3.12 \mathrm{E}-01$ \\
\hline
\end{tabular}

\begin{tabular}{|l|r|r|r|r|r|r|}
\hline & \multicolumn{2}{|c|}{ SDS, high - Ads } & \multicolumn{2}{c|}{ SDS, high - Elute } & \multicolumn{2}{c|}{ Nal Ads } \\
\hline Chirality $\mathbf{( n , m )}$ & $\# S W N T / m L$ & fractional purity & \#SWNT/mL & fractional purity & $\# S W N T / m L$ & fractional purity \\
\hline $\mathbf{( 1 0 , 2 )}$ & $4.54 \mathrm{E}+09$ & $3.96 \mathrm{E}-03$ & $1.51 \mathrm{E}+10$ & $6.42 \mathrm{E}-02$ & $3.43 \mathrm{E}+07$ & $3.09 \mathrm{E}-05$ \\
\hline $\mathbf{( 7 , 5 )}$ & $9.05 \mathrm{E}+10$ & $7.90 \mathrm{E}-02$ & $2.72 \mathrm{E}+10$ & $1.16 \mathrm{E}-01$ & $8.50 \mathrm{E}+10$ & $7.64 \mathrm{E}-02$ \\
\hline $\mathbf{( 6 , 5 )}$ & $5.02 \mathrm{E}+11$ & $4.38 \mathrm{E}-01$ & $1.02 \mathrm{E}+11$ & $4.33 \mathrm{E}-01$ & $5.22 \mathrm{E}+11$ & $4.70 \mathrm{E}-01$ \\
\hline $\mathbf{( 7 , 3 )}$ & $1.61 \mathrm{E}+11$ & $1.41 \mathrm{E}-01$ & $2.60 \mathrm{E}+10$ & $1.10 \mathrm{E}-01$ & $9.94 \mathrm{E}+10$ & $8.93 \mathrm{E}-02$ \\
\hline $\mathbf{( 8 , 3 )}$ & $2.32 \mathrm{E}+07$ & $2.02 \mathrm{E}-05$ & $2.22 \mathrm{E}+10$ & $9.43 \mathrm{E}-02$ & $8.84 \mathrm{E}+09$ & $7.95 \mathrm{E}-03$ \\
\hline$(\mathbf{9 , 1})$ & $6.99 \mathrm{E}+09$ & $6.10 \mathrm{E}-03$ & $1.34 \mathrm{E}+09$ & $5.71 \mathrm{E}-03$ & $1.53 \mathrm{E}+10$ & $1.38 \mathrm{E}-02$ \\
\hline $\mathbf{( 6 , 4 )}$ & $3.81 \mathrm{E}+11$ & $3.32 \mathrm{E}-01$ & $4.17 \mathrm{E}+10$ & $1.77 \mathrm{E}-01$ & $3.81 \mathrm{E}+11$ & $3.43 \mathrm{E}-01$ \\
\hline
\end{tabular}

\begin{tabular}{|l|r|r|r|r|r|r|}
\hline & \multicolumn{2}{|c|}{ Nal Elute } & \multicolumn{2}{c|}{ NaBr Ads } & \multicolumn{2}{c|}{ NaBr Elute } \\
\hline Chirality $\mathbf{( n , m )}$ & \#SWNT/mL & fractional purity & \#SWNT/mL & fractional purity & \#SWNT/mL & fractional purity \\
\hline $\mathbf{( 1 0 , 2 )}$ & $2.67 \mathrm{E}+08$ & $3.63 \mathrm{E}-04$ & $3.53 \mathrm{E}+07$ & $3.38 \mathrm{E}-05$ & $1.99 \mathrm{E}+10$ & $2.25 \mathrm{E}-02$ \\
\hline $\mathbf{( 7 , 5 )}$ & $9.93 \mathrm{E}+10$ & $1.35 \mathrm{E}-01$ & $6.13 \mathrm{E}+10$ & $5.88 \mathrm{E}-02$ & $1.26 \mathrm{E}+11$ & $1.43 \mathrm{E}-01$ \\
\hline $\mathbf{( 6 , 5 )}$ & $3.02 \mathrm{E}+11$ & $4.10 \mathrm{E}-01$ & $4.98 \mathrm{E}+11$ & $4.77 \mathrm{E}-01$ & $3.71 \mathrm{E}+11$ & $4.20 \mathrm{E}-01$ \\
\hline $\mathbf{( 7 , 3 )}$ & $5.81 \mathrm{E}+10$ & $7.88 \mathrm{E}-02$ & $9.78 \mathrm{E}+10$ & $9.37 \mathrm{E}-02$ & $8.66 \mathrm{E}+10$ & $9.80 \mathrm{E}-02$ \\
\hline $\mathbf{( 8 , 3 )}$ & $1.96 \mathrm{E}+09$ & $2.67 \mathrm{E}-03$ & $3.12 \mathrm{E}+07$ & $2.99 \mathrm{E}-05$ & $3.11 \mathrm{E}+07$ & $3.52 \mathrm{E}-05$ \\
\hline$(\mathbf{9 , 1})$ & $2.30 \mathrm{E}+10$ & $3.13 \mathrm{E}-02$ & $6.99 \mathrm{E}+09$ & $6.70 \mathrm{E}-03$ & $3.56 \mathrm{E}+10$ & $4.03 \mathrm{E}-02$ \\
\hline $\mathbf{( 6 , 4 )}$ & $2.52 \mathrm{E}+11$ & $3.42 \mathrm{E}-01$ & $3.80 \mathrm{E}+11$ & $3.64 \mathrm{E}-01$ & $2.44 \mathrm{E}+11$ & $2.76 \mathrm{E}-01$ \\
\hline
\end{tabular}

\begin{tabular}{|l|r|r|r|r|r|r|}
\hline & \multicolumn{2}{|c|}{ NaCl Ads } & \multicolumn{2}{c|}{ NaCl Elute } & \multicolumn{2}{c|}{ NaF Ads } \\
\hline Chirality $(\mathbf{n}, \mathbf{m})$ & \#SWNT/mL & fractional purity & \#SWNT/mL & fractional purity & \#SWNT/mL & fractional purity \\
\hline $\mathbf{( 1 0 , 2 )}$ & $7.92 \mathrm{E}+07$ & $7.20 \mathrm{E}-05$ & $2.00 \mathrm{E}+10$ & $2.14 \mathrm{E}-02$ & $2.91 \mathrm{E}+07$ & $2.85 \mathrm{E}-05$ \\
\hline $\mathbf{( 7 , 5 )}$ & $8.34 \mathrm{E}+10$ & $7.57 \mathrm{E}-02$ & $1.29 \mathrm{E}+11$ & $1.38 \mathrm{E}-01$ & $7.01 \mathrm{E}+10$ & $6.88 \mathrm{E}-02$ \\
\hline $\mathbf{( 6 , 5 )}$ & $5.01 \mathrm{E}+11$ & $4.55 \mathrm{E}-01$ & $3.94 \mathrm{E}+11$ & $4.21 \mathrm{E}-01$ & $4.53 \mathrm{E}+11$ & $4.45 \mathrm{E}-01$ \\
\hline $\mathbf{( 7 , 3 )}$ & $1.27 \mathrm{E}+11$ & $1.16 \mathrm{E}-01$ & $9.51 \mathrm{E}+10$ & $1.02 \mathrm{E}-01$ & $1.29 \mathrm{E}+11$ & $1.27 \mathrm{E}-01$ \\
\hline $\mathbf{( 8 , 3 )}$ & $1.91 \mathrm{E}+07$ & $1.73 \mathrm{E}-05$ & $2.97 \mathrm{E}+07$ & $3.17 \mathrm{E}-05$ & $2.17 \mathrm{E}+07$ & $2.13 \mathrm{E}-05$ \\
\hline $\mathbf{( 9 , 1 )}$ & $3.96 \mathrm{E}+09$ & $3.60 \mathrm{E}-03$ & $3.59 \mathrm{E}+10$ & $3.84 \mathrm{E}-02$ & $2.63 \mathrm{E}+09$ & $2.59 \mathrm{E}-03$ \\
\hline $\mathbf{( 6 , 4 )}$ & $3.86 \mathrm{E}+11$ & $3.50 \mathrm{E}-01$ & $2.62 \mathrm{E}+11$ & $2.80 \mathrm{E}-01$ & $3.64 \mathrm{E}+11$ & $3.57 \mathrm{E}-01$ \\
\hline
\end{tabular}

\begin{tabular}{|l|r|r|r|r|r|r|}
\hline & \multicolumn{2}{|c|}{ NaF Elute } & \multicolumn{2}{c|}{ Urea, low - Ads } & \multicolumn{2}{c|}{ Urea, low - Elute } \\
\hline Chirality $(\mathbf{n}, \mathbf{m})$ & \#SWNT/mL & fractional purity & $\# S W N T / m L$ & fractional purity & $\# S W N T / m L$ & fractional purity \\
\hline $\mathbf{( 1 0 , 2 )}$ & $5.40 \mathrm{E}+09$ & $6.03 \mathrm{E}-03$ & $1.41 \mathrm{E}+10$ & $1.27 \mathrm{E}-02$ & $3.14 \mathrm{E}+09$ & $4.44 \mathrm{E}-02$ \\
\hline $\mathbf{( 7 , 5 )}$ & $9.61 \mathrm{E}+10$ & $1.07 \mathrm{E}-01$ & $1.02 \mathrm{E}+11$ & $9.20 \mathrm{E}-02$ & $1.04 \mathrm{E}+10$ & $1.48 \mathrm{E}-01$ \\
\hline $\mathbf{( 6 , 5 )}$ & $4.22 \mathrm{E}+11$ & $4.72 \mathrm{E}-01$ & $4.75 \mathrm{E}+11$ & $4.29 \mathrm{E}-01$ & $2.96 \mathrm{E}+10$ & $4.18 \mathrm{E}-01$ \\
\hline $\mathbf{( 7 , 3 )}$ & $7.24 \mathrm{E}+10$ & $8.09 \mathrm{E}-02$ & $1.50 \mathrm{E}+11$ & $1.36 \mathrm{E}-01$ & $7.42 \mathrm{E}+09$ & $1.05 \mathrm{E}-01$ \\
\hline $\mathbf{( 8 , 3 )}$ & $2.25 \mathrm{E}+09$ & $2.51 \mathrm{E}-03$ & $1.33 \mathrm{E}+07$ & $1.20 \mathrm{E}-05$ & $3.57 \mathrm{E}+09$ & $5.05 \mathrm{E}-02$ \\
\hline $\mathbf{( 9 , 1 )}$ & $2.20 \mathrm{E}+10$ & $2.46 \mathrm{E}-02$ & $3.52 \mathrm{E}+09$ & $3.18 \mathrm{E}-03$ & $1.68 \mathrm{E}+09$ & $2.37 \mathrm{E}-02$ \\
\hline $\mathbf{( 6 , 4 )}$ & $2.75 \mathrm{E}+11$ & $3.07 \mathrm{E}-01$ & $3.63 \mathrm{E}+11$ & $3.28 \mathrm{E}-01$ & $1.49 \mathrm{E}+10$ & $2.11 \mathrm{E}-01$ \\
\hline
\end{tabular}

Table S7: SWCNT concentration and relative abundance of each chiral species for data presented in Figure $\mathbf{7}$ of the main text. 


\section{S3. Comparative Spectral Fitting and Deconvolution Analysis}

It is necessarily important that the spectral deconvolution of measured absorbance spectra be an accurate reflection of the data presented. In the present work, we used an in-house written Mathematica program to quantitively ascertain the number concentration and therefore relative purity of SWCNT suspensions to gain insight into the mechanistic aspects of SWNCT-gel interactions. Briefly, the program involved an amorphous carbon, linear background and the identification of a least squares best-fit between a given experimental absorbance spectrum and the sum of the individual chiral contributions. Because we exclusively used raw powder containing $\geq 95 \%$ semiconducting SWCNT and fit exclusively within the E11 region of the spectrum, we considered only the $(10,2),(7,5),(6,5),(7,3),(8,3),(9,1)$, and $(6,4)$ species for fitting. With regard to our fitting procedure, all considered species had salient E11 peaks and thus only these were fitted and were done so with Lorentzian line shapes. A more in-depth discussion of this approach is described in the supporting information of our previous work. ${ }^{1}$ There do, however, also exist other fitting methods that are robust and thorough. For example, Flavel et al. presented a comprehensive SWCNT fitting approach with freely distributed corresponding MATLAB code. ${ }^{4}$ In this section, we provide a side-by-side comparison of our fitting method and that detailed by Flavel et al. The

This Work

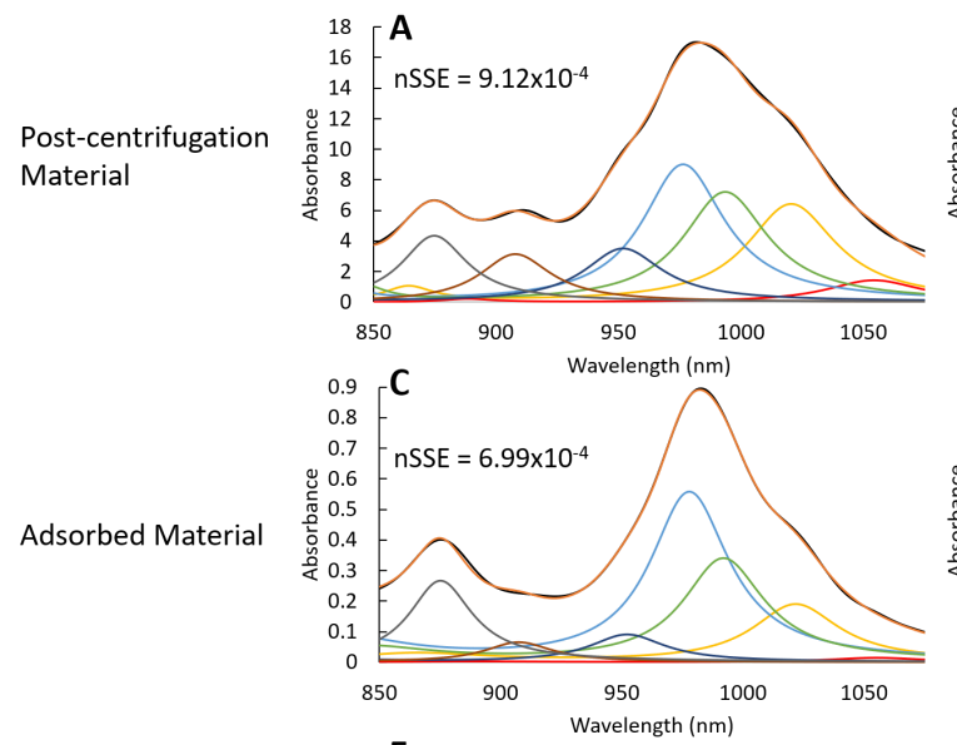

Post-centrifugation Material

Eluted Material

Recorded

Spectrum Spectrum

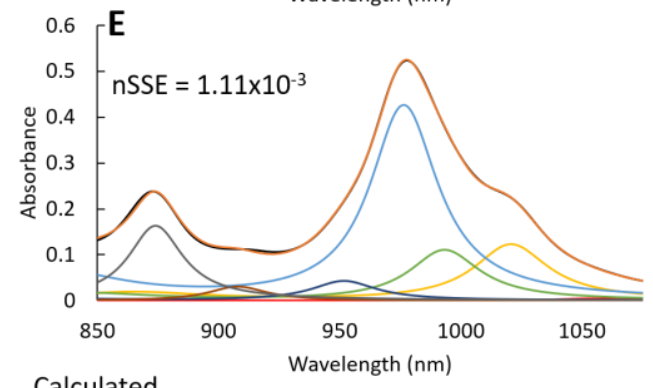

$$
(10,2)
$$

$(7,5)$
ACS Omega 2017, 2, 3, 1163-1171
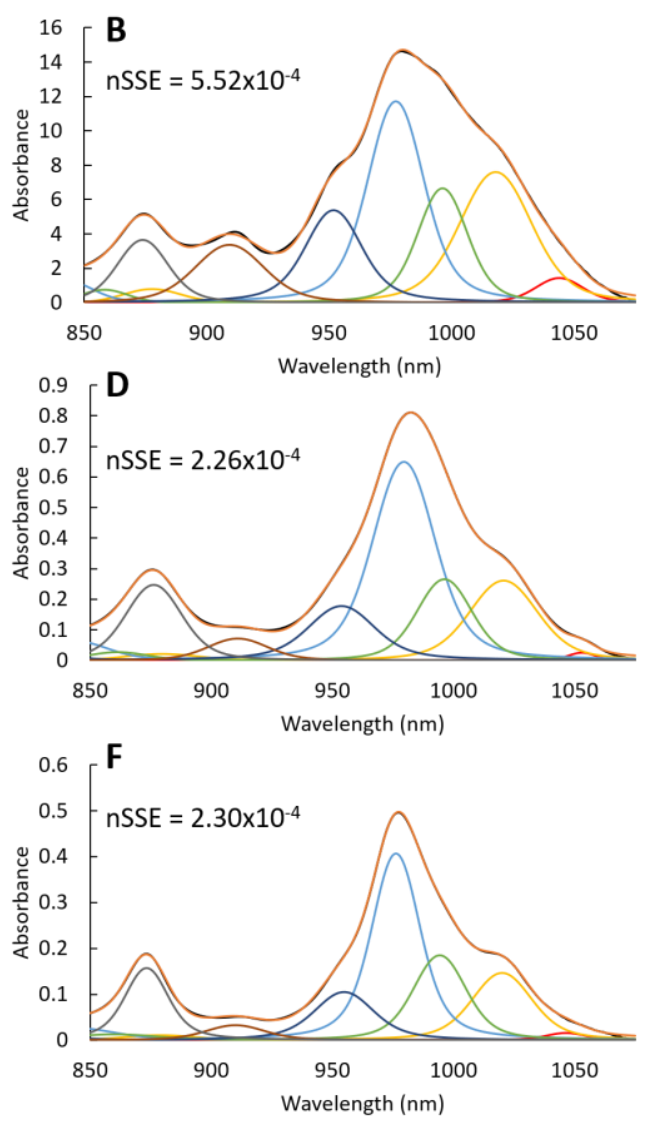

$(7,3)$
$(8,3)$

$(9,1)$
$(6,4)$

Figure S1. (A) Post-centrifugation, (C) adsorbed, and (E) eluted SWCNT with Lorentzian line shapes using the spectral fitting present in this work. Corresponding (B) Post-centrifugation, (D) adsorbed, and (F) eluted SWCNT with Voight line shapes using the fitting program by Flavel et al. ${ }^{4}$ On each spectrum is the normalized sum of squared errors (nSSE) which indicate the goodness of the fit. 
comparisons were done using the same experimental absorbance spectra from this work, as presented in Fig. 1B, Fig. 1E, and Fig. 1F of the main text.

The deconvoluted absorbance spectra of post-centrifugation, adsorbed, and eluted SWCNT are presented in Fig. S1. The left-hand side of this figure are the fitted spectra using the approach utilized within this work (Mathematica), while the right-hand side are the same experimental spectra fitted with the program distributed with the ACS Omega publication (MATLAB). The deconvoluted spectra in obtained via Mathematica strictly use Lorentzian line shapes, while the MATLAB program affords fitting using Gaussian, Lorentzian, or Voight line shapes. There are more free parameters available to users with Flavel's program. In using the MATLAB program, we found that Voight line shapes yielded the best fit to each of these spectra. This is evident by the normalized sum of squared errors (nSSE) values presented alongside each spectrum in Fig. S1. We found the nSSE parameter particularly important and while the MATLAB program inherently provides this, we found it necessary to do the same for our spectra. In general, the MATLAB program provides nSSE closer to zero, indicating better fits. However the nSSE values for our Mathematica program are generally the same in magnitude, save the eluted material. Nevertheless, both programs provide more than reasonable deconvolutions within the $\mathrm{E}_{11}$ region.

From the spectral deconvolution, it is possible to obtain the number concentration and hence the relative abundance of chiralities present in a sample. Typically, we use the $E_{11}$ peak height for each chirality, the chirality dependent absorbance cross section, and the chirality dependent number of carbon atoms as a function of tube length. Similarly, the MATLAB program uses known parameters of peak height, path length, and molar absorptivity to obtain relative concentration. In Fig. S2 are the total number of SWCNT in the post-centrifugation, adsorbed, and eluted material and the fractional purity of the fitted chiralities as determined from each of the three spectra shown in Fig. S1. The MATLAB program accounts for an average of $15 \%$ more SWCT in post-centrifugation, adsorbed, and eluted samples, as seen in Figures S2A, S2B, and S2C.

Differences in the deconvoluted absorbance spectra and resultant total SWCNT and chirality fractional purity between the two methods can be traced back to variations in best-fit approach. The origin of these differences are results of the peak heights, FWHM values, and centers associated with the $E_{11}$ and
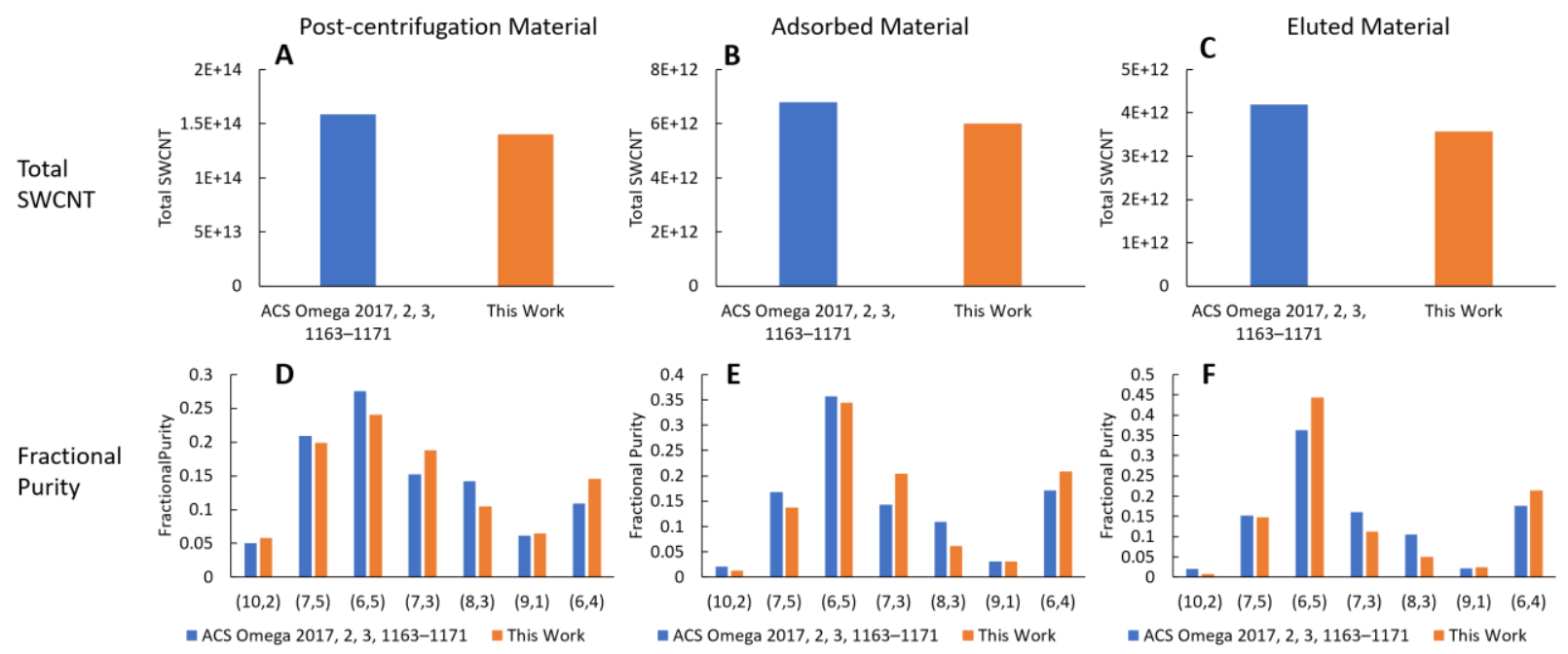

Figure S2. The SWCNT number analysis from the spectral fitting data from two methods. The total SWCNT in (A) post-centrifugation, (B) adsorbed, and (C) eluted materials. The relative fractional purities of chiralities in (D) postcentrifugation, (E) adsorbed, and (F) eluted materials. The blue bars indicate use of the program by Flavel et al., while the orange bars result from our spectral fitting methodology. 
corresponded PSB (phonon sideband). The differences in these are presented in Fig. S3. It should be noted that this quantitative analysis is only for the post-centrifugation SWCNT material. Our method constrains crucial parameters of peak height, FWHM, and centers. This results in $\mathrm{E}_{11} / \mathrm{PSB}$ height in Fig. S3C, FWHM values of the $E_{11}$ and PSB in Figs. S3 $A$ and $B$, respectively, and PSB relative to the $E_{11}$ in Fig. S3I, in which the FWHM for the $E_{11}$ and PSB remain constant. While the FWHM for the $E_{11}$ and PSB with our method are larger than that in the MATLAB approach, this is compensated for in total area under the curve by generally having smaller peak height in our program, which is ultimately manifested in less overall SWCNT, Fig. S2 A-C.

In conducting a direct comparison between the method used for this work and the method by Flavel et al., ${ }^{4}$ we believe our spectral fitting to be an accurate representation of the trends seen in the main text. Resulting trends in chirality-specific SWCNT-gel interactions remain consistent regardless of the method used.
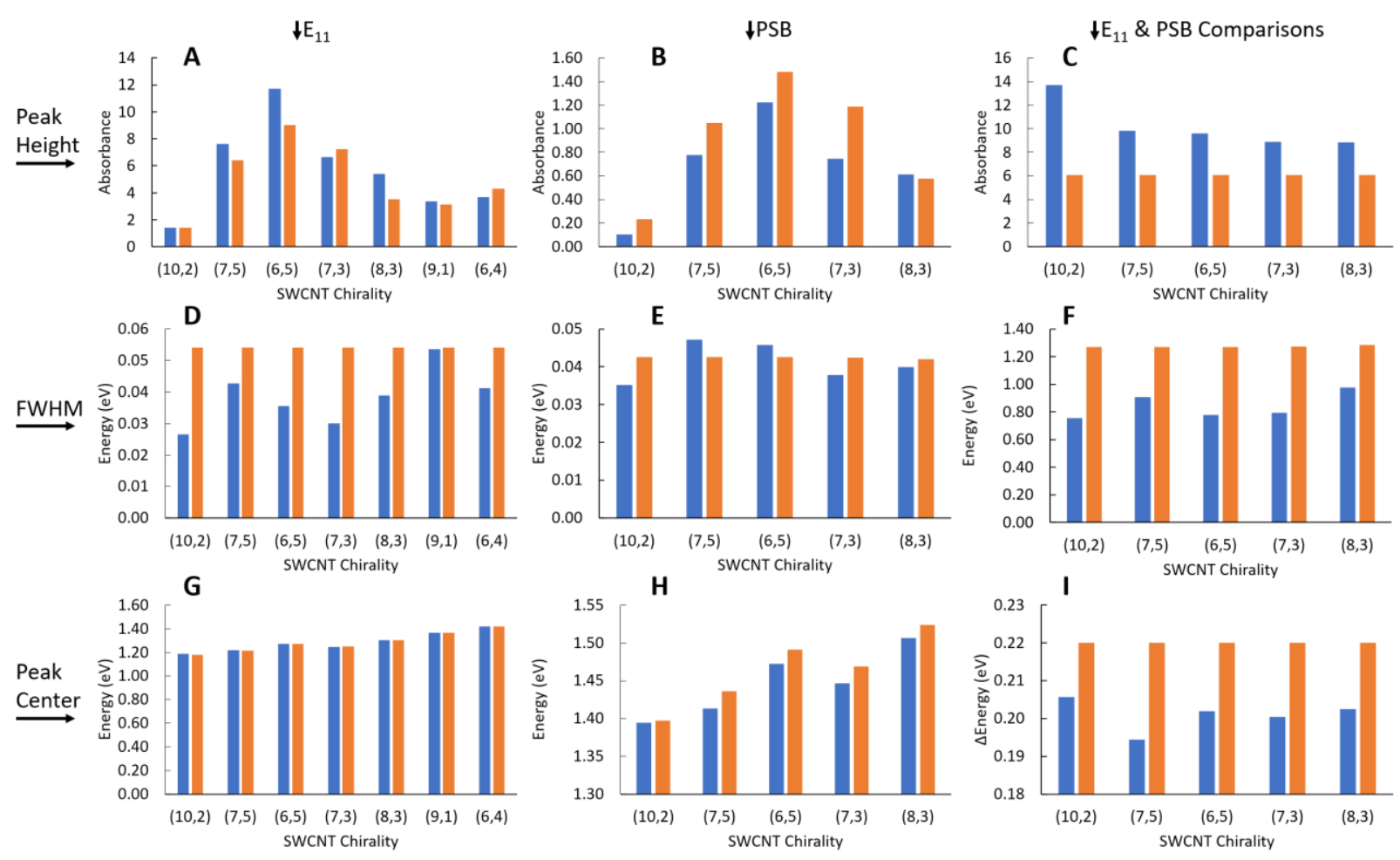

ACS Omega 2017, 2, 3, 1163-1171 This Work

Figure S3. Pertinent chirality-specific parameters associated with spectral fitting for post-centrifugation material. (A) $E_{11}$ peak height, (B) PSB peak height, and (C) peak height ratios of $E_{11}$ relative to PSB. (D) $E_{11}$ FWHM, (E) PSB FWHM, and (F) FWHM ratios of $E_{11}$ relative to PSB. (G) $E_{11}$ peak energetic centers, (H) PSB peak energetic centers, and (I) Relative energetic difference between $\mathrm{E}_{11}$ and corresponding PSB centers. 


\section{S4. Intermediate Data Pertaining to the Adsorption of SWCNT to Sephacryl at Various SDS Concentration and Elution thereof.}

To investigate the potential for SDS-surfactant mediated process efficiency, a series of SWCNTSephacryl interactions were conducted at SDS concentrations ranging from $26 \mathrm{mM}$ to $105 \mathrm{mM}$ during the adsorption event. Major findings and the implications of these data are discussed in the main text of this work, while additional data relating to the \# of SWCNT adsorbed and desorbed at each SDS concentration are provided here.

Absorbance traces of SWCNT suspensions introduced to Sephacryl S200 gel beds are provided in Figure S4, with SWCNT content ranging from $6.4 \mathrm{E}+12$ to $7.4 \mathrm{E}+12 \mathrm{SWCNT} / \mathrm{mL}$ with a total applied volume of $+4.0 \mathrm{~mL}$. Traces corresponding to the SWCNT eluted from each gel are provided in Figure S5, while quantification of the \# of SWCNT adsorbed and eluted at each SDS concentration are shown in Figure S6.

It is important to note that the data acquired from this set of investigations was employed to determine a standard number of SWCNT adsorption sites per volume of Sephacryl hydrogel. Specifically, the number of SWCNT adsorbed to each gel bed (absorbance traces provided in Figure $\mathbf{2}$ of the main text) was averaged over the range of 26-79 mM SDS, across which the number of SWCNT adsorbed was relatively constant (average of 6.7E+12 SWCNT per $150 \mu \mathrm{L}$ of settled Sephacryl S200 hydrogel, Figure S3). As such, the standardized \# of gel binding sites for this work was determined to be 4.5E+10 SWCNT per $\mu \mathrm{L}$ of settled S200 gel. This corresponds to $4.2 \mathrm{E}+10$ SWCNT per $\mathrm{cm}^{2}$ of Sephacryl S200 surface area, a value that is applied throughout this work to calculate the number of available hydrogel binding sites.

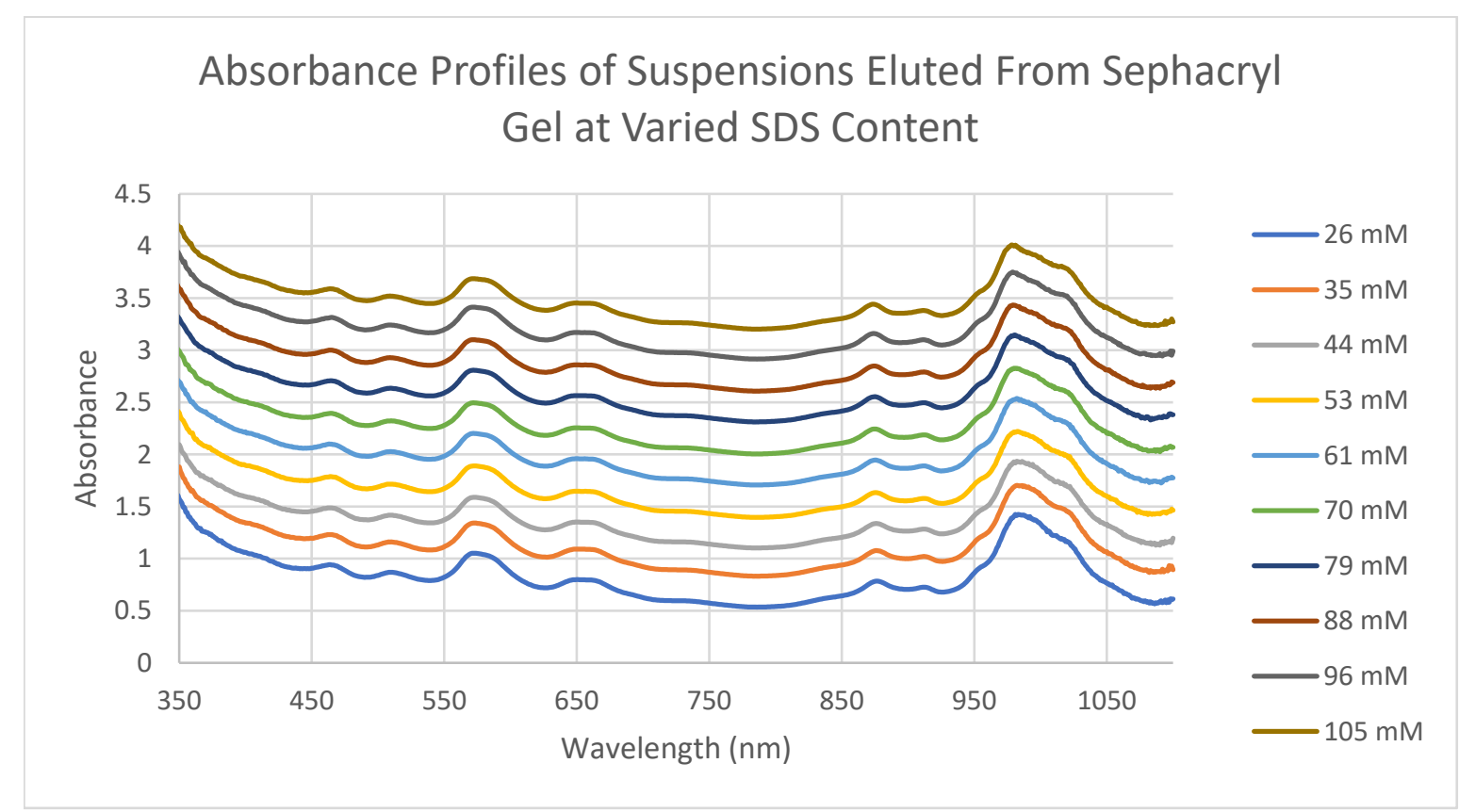

Figure S4: Offset absorbance traces of SWCNT suspensions introduced to gel columns for data presented in Figure 2 of the main body. The \# of SWCNT in each suspension ranges from $6.4 \mathrm{E}+12$ to 7.4E+12 SWCNT/mL. 


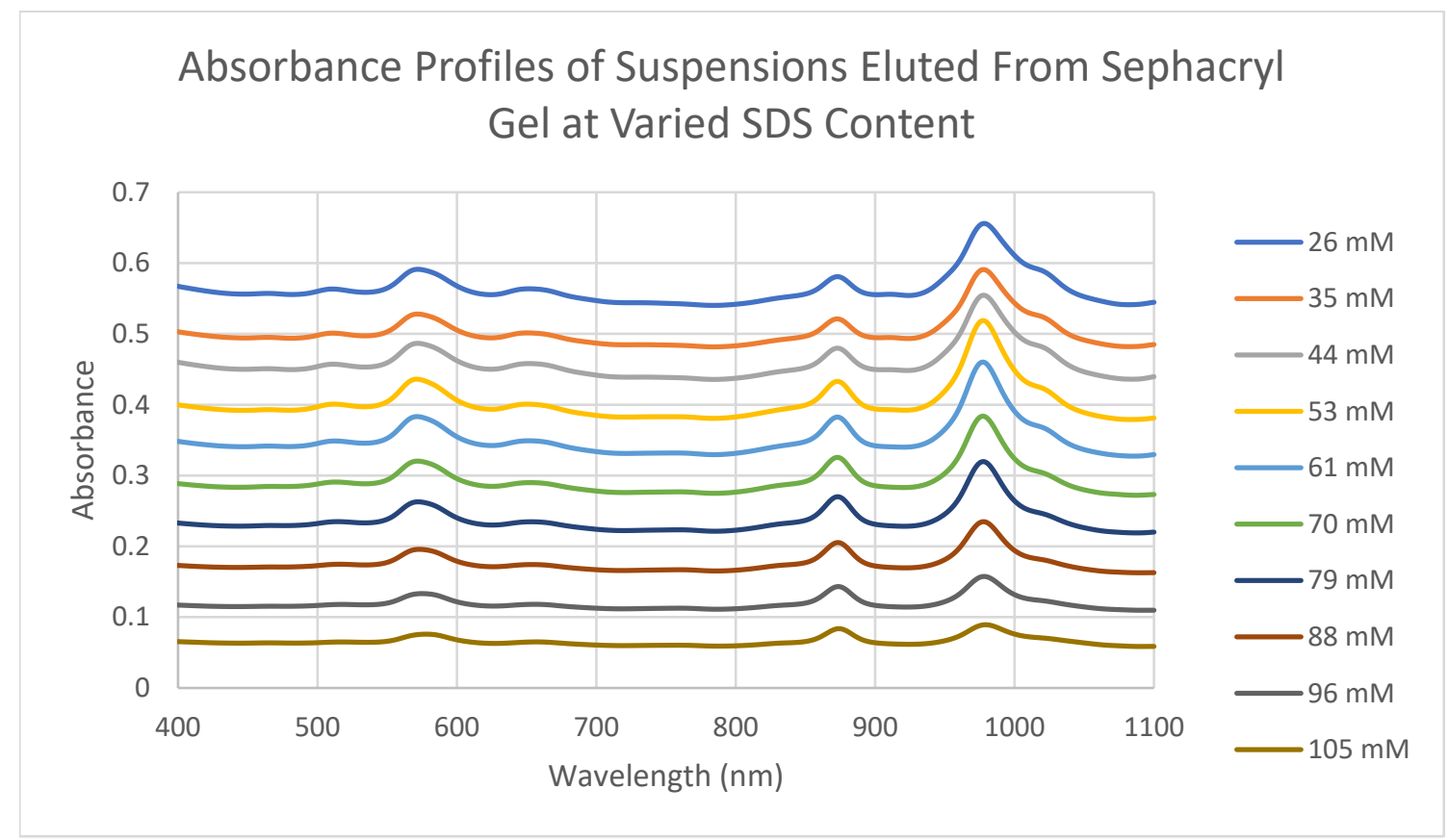

Figure S5: Offset absorbance traces of SWCNT suspensions eluted from gel columns for data presented in Figure 2 of the main body.

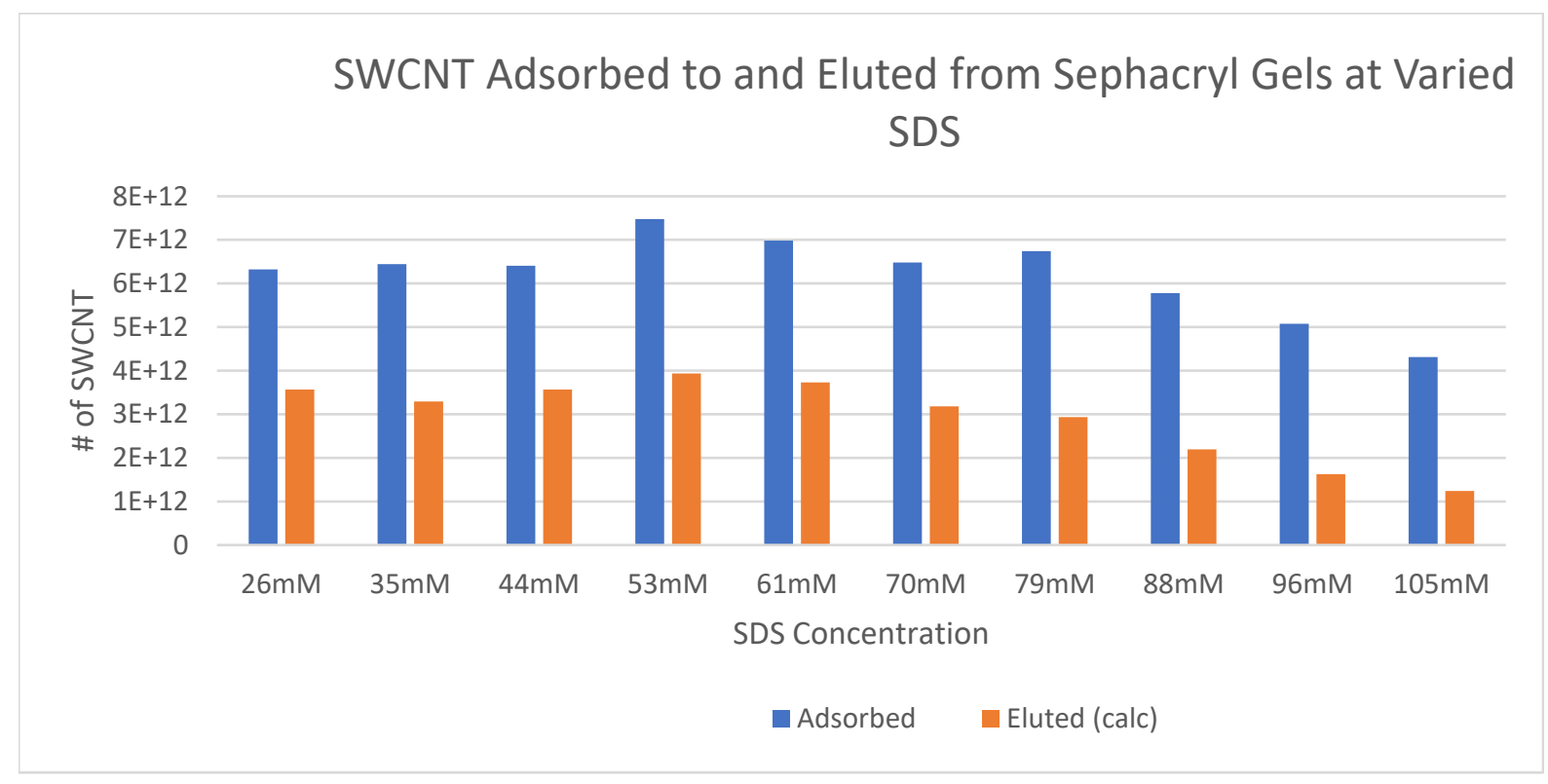

Figure S6: Absolute number of $300 \mathrm{~nm}$ length SWCNT adsorbed to and eluted from Sephacryl gels at varied SDS Concentration for data presented in Figure $\mathbf{2}$ of the main body. 


\section{S5. Intermediate Data Pertaining to the Adsorption of SWCNT to Sephacryl at Various SWCNT Concentration and Elution thereof.}

To explore the potential for SWCNT of identical chiral type to exist in either a ReBS or an IrBS, a series of SWCNT-Sephacryl interactions were performed at loading ratio $\rho$ of 1.7 to 4.5 . While relevant findings from these experiments and their implications for the generation of an ReBS and IrBS are included in the main body of this work, this section provides additional spectroscopic data relating to the SWCNT suspensions introduced to each gel bed, Figure S7, and the corresponding spectra of SWCNT material flowed through those gels, Figure $\mathbf{S 8}$.

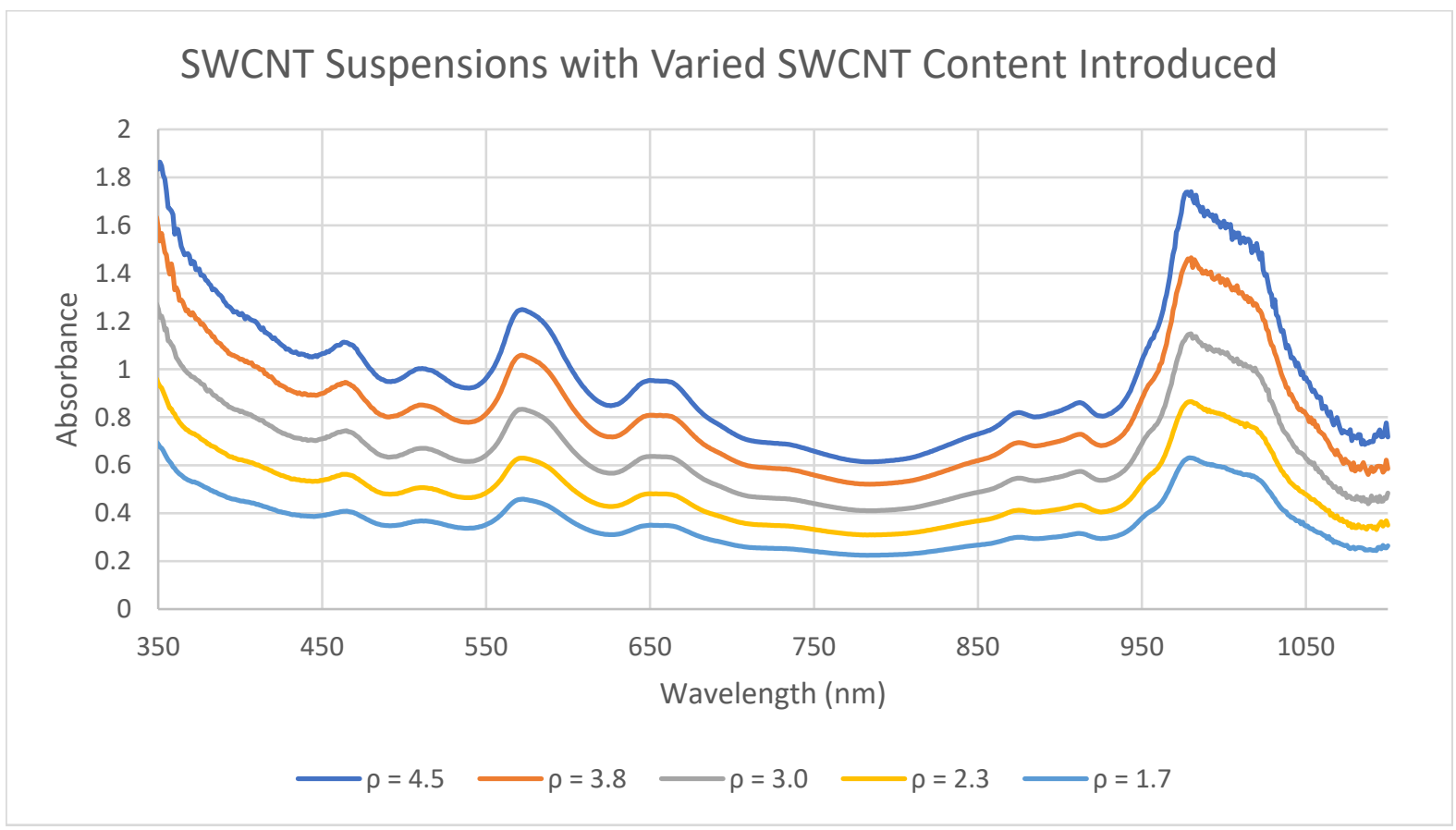

Figure S7: Absorbance traces of SWCNT suspensions with varied SWCNT content which were introduced to gel beds for collection of data presented in Figure $\mathbf{3}$ in the main body. 


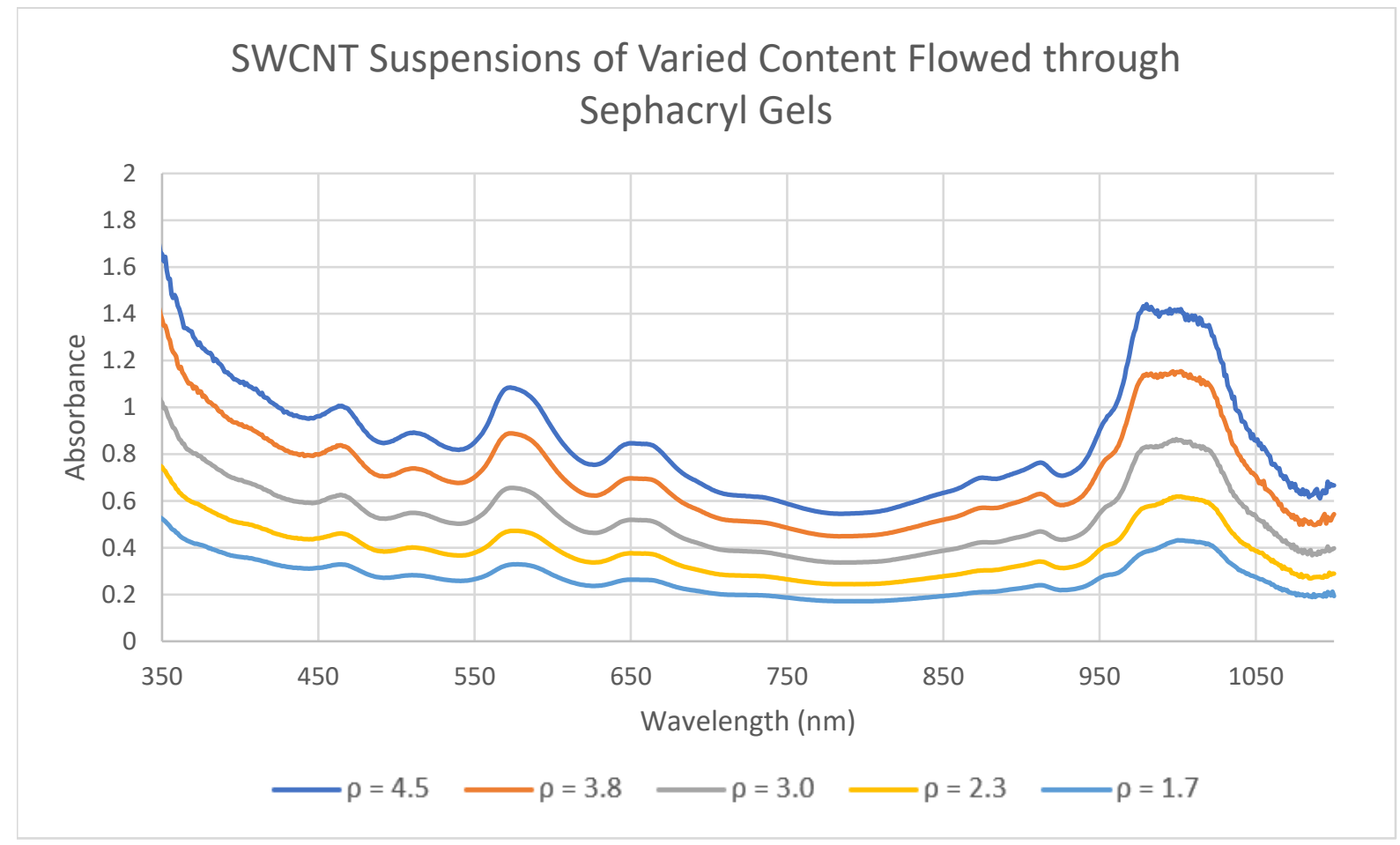

Figure S8: Absorbance traces of SWCNT suspensions with varied SWCNT content which were flowedthrough gel beds for collection of data presented in Figure $\mathbf{3}$ in the main body. 


\section{S6. References}

(1) Watts, B. P.; Barbee, C. H.; Tvrdy, K. Exploiting the Physiochemical Interactions between SingleWalled Carbon Nanotubes and Hydrogel Microspheres To Afford Chirally Pure Nanotubes. ACS Appl. Nano Mat. 2019, 2 (6), 3615-3625.

(2) Dolan, M.; Watts, B. P.; Tvrdy, K. Tailored Synthesis of Hydrogel Media for Chirality Separation of Single Walled Carbon Nanotubes. Carbon 2021, 171, 597-609.

(3) Zanoni, S.; Watts, B. P.; Tvrdy, K. Single-Walled Carbon Nanotube Chiral Selectivity Exhibited by Commercially Available Hydrogels of Varying Composition. ACS Appl. Mater. Interfaces 2021, 13 (28), 33635-33643.

(4) Pfohl, M.; Tune, D. D.; Graf, A.; Zaumseil, J.; Krupke, R.; Flavel, B. S. Fitting Single-Walled Carbon Nanotube Optical Spectra. ACS Omega 2017, 2 (3), 1163-1171. 